Review

\title{
The Role of Interleukins in Colorectal Cancer
}

\author{
Jingjing Li1 ${ }^{*}$, Ling Huang1 ${ }^{*}$, Hanzhang Zhao1, Yuheng Yan¹, Jing $\mathrm{Lu}^{2,3,4}{ }^{\bowtie}$ \\ 1. Department of Clinical Medicine, Grade 2017, School of Basic Medical Sciences, Zhengzhou University, Zhengzhou 450001, Henan, China. \\ 2. Department of Pathophysiology, School of Basic Medical Sciences, Zhengzhou University, Zhengzhou 450001, Henan, China. \\ 3. Collaborative Innovation Center of Henan Province for Cancer Chemoprevention, Zhengzhou University, Zhengzhou 450001, Henan, China. \\ 4. State Key Laboratory of Esophageal Cancer Prevention and Treatment, Zhengzhou University, Zhengzhou 450001, Henan, China. \\ ${ }^{*}$ Contributed equally to the manuscript. \\ $\square$ Corresponding author: Dr Jing Lu, Department of Pathophysiology, School of Basic Medical Sciences, Zhengzhou University, No.100 Science Road, \\ Zhengzhou 450001, Henan, China; E-mail: lujing@zzu.edu.cn.
}

(C) The author(s). This is an open access article distributed under the terms of the Creative Commons Attribution License (https://creativecommons.org/licenses/by/4.0/). See http://ivyspring.com/terms for full terms and conditions.

Received: 2020.04.04; Accepted: 2020.05.30; Published: 2020.06.14

\begin{abstract}
Despite great progress has been made in treatment strategies, colorectal cancer (CRC) remains the predominant life-threatening malignancy with the feature of high morbidity and mortality. It has been widely acknowledged that the dysfunction of immune system, including aberrantly expressed cytokines, is strongly correlated with the pathogenesis and progression of colorectal cancer. As one of the most well-known cytokines that were discovered centuries ago, interleukins are now uncovering new insights into colorectal cancer therapy. Herein, we divide currently known interleukins into 6 families, including IL-1 family, IL-2 family, IL-6 family, IL-8 family, IL-10 family and IL-17 family. In addition, we comprehensively reviewed the oncogenic or antitumour function of each interleukin involved in CRC pathogenesis and progression by elucidating the underlying mechanisms. Furthermore, by providing interleukins-associated clinical trials, we have further driven the profound prospect of interleukins in the treatment of colorectal cancer.
\end{abstract}

Key words: Colorectal cancer; Interleukins; Molecular mechanism; Clinical therapy

\section{Introduction}

Colorectal cancer $(\mathrm{CRC})$ is the third most common cancer and the fourth leading cause of cancer-related death worldwide [1]. Currently, the main treatment for CRC is surgical resection, supplemented by systemic chemotherapy and local pelvic radiotherapy [2]. However, CRC treatments still face enormous challenges. In 2018, 1.8 million new cases were diagnosed, and one-third of these new patients had metastatic cancer [3]. Although, in developed countries, the use of early screening has significantly increased the 5-year survival rate for patients with CRC [4], the latest data show that the prevalence of early-onset CRC is increasing, especially the incidence rate in young people with rectal cancer [3]. The study also shows that the incidence rate of CRC in the United States is expected to increase by $90 \%$ by 2030 . In such a tough situation, people are eager to seek new therapeutic strategies.

Interleukins (ILs) can be divided into several families with more than 40 subfamily members. They can interact with a variety of cells that alter the immune system and act on a wide range of cancers. In the past several years, ILs have attracted substantial attention because of their distinct roles in CRC (Table 1) that provide a new and promising strategy for CRC. In general, ILs facilitate CRC by promoting tumorigenesis [5], tumour growth [6], angiogenesis [7], and cancer cell invasion and metastasis [7] and inhibit CRC via complex pathways. In addition, some clinical trials in progress are expected to lead to a breakthrough in the treatment of CRC. In this review, we summarize the distinct roles of the diverse IL families with an impact in CRC and emphasize their internal mechanisms and clinical applications by focusing on recent studies in the hope of providing some useful inspiration for continued pursuit of yet unrevealed mechanisms and further clinical research. 
Table 1. Summary of the interleukin families and the roles in CRC

\begin{tabular}{|c|c|c|c|c|c|}
\hline Interleukin Family & Cytokine & Receptor & Functional effect in CRC & Expression Patterns & Reference \\
\hline \multirow[t]{8}{*}{ IL-1 FAMILY } & IL-1a & IL-1R1 IL-1R2 & promote metastasis and the chemosensitivity & $\uparrow$ & {$[11][14]$} \\
\hline & IL-1 $\beta$ & IL-1R1 IL-1R2 & $\begin{array}{l}\text { promote the proliferation of colon cancer cells, } \\
\text { promote tumorigenesis, alter the tumour } \\
\text { microenvironment }\end{array}$ & $\uparrow$ & [11] [25] \\
\hline & IL-1Ra & IL-1R1 IL-1R2 & confusing & $\uparrow$ & [10] \\
\hline & IL-33 & IL-1R4 (ST2) & $\begin{array}{l}\text { alter tumour microenvironment, promote } \\
\text { angiogenesis, enhance colon cancer cell stemness, } \\
\text { suppress sporadic colon cancer, maintain } \\
\text { intestinal microbiota homeostasis }\end{array}$ & $\uparrow$ & {$[7,12][37-40]$} \\
\hline & IL-18 & IL-1R5 (IL-18Ra IL-18R $\beta$ ) & act on NK cells, improve intestinal barrier integrity & $\downarrow$ & {$[11][30]$} \\
\hline & IL-36a & IL-1R6 & antitumour & -- & [34] \\
\hline & IL-36y & IL-1R6 & promote inflammatory immune infiltrates & -- & {$[34]$} \\
\hline & IL-37 & IL-1R5 & $\begin{array}{l}\text { inhibit the development of colon cancer cells by } \\
\text { inhibiting } \beta \text {-catenin }\end{array}$ & $\downarrow$ & [33] [35] \\
\hline \multirow[t]{6}{*}{ IL-2 FAMILY } & IL-2 & 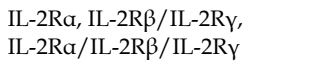 & antitumour & -- & [43] [44] \\
\hline & IL-4 & $\begin{array}{l}\text { TypeI (IL-4Ra/үc) and Type II } \\
\text { (IL-4Ra/IL-13Ra1) }\end{array}$ & $\begin{array}{l}\text { promote EMT, proliferation, invasion and } \\
\text { metastasis }\end{array}$ & $\uparrow$ & {$[51][52]$} \\
\hline & IL-7 & IL-7R (IL-7Ra/ үc) & promote metastasis & $\uparrow$ & {$[49][50]$} \\
\hline & IL-9 & IL-9R (IL-9Ra/үc) & antitumour & $\downarrow$ & {$[45][58]$} \\
\hline & IL-15 & IL-15R (IL-15Ra/IL-15R $\beta / \gamma c)$ & $\begin{array}{l}\text { inhibit proliferation and angiogenesis, promote } \\
\text { apoptosis }\end{array}$ & -- & [46] \\
\hline & IL-21 & heterodimers of IL-21R and $\gamma c$ & confusing & $\uparrow$ & {$[47-48][59-61]$} \\
\hline \multirow[t]{2}{*}{ IL-6 FAMILY } & IL-6 & gp130 IL-6R & $\begin{array}{l}\text { promote mitosis, proliferation, metastasis, } \\
\text { migration, angiogenesis and make a } \\
\text { microenvironment which is good for the } \\
\text { metastasis }\end{array}$ & $\uparrow$ & $\begin{array}{l}\text { Knupfer H, Preiss R. International } \\
\text { journal of colorectal disease. } \\
2010 ; 25(2): 135-40\end{array}$ \\
\hline & IL-11 & gp130 IL-11Ra & facilitate the proliferation of CRC & $\uparrow$ & $\begin{array}{l}\text { Putoczki TL, et al. Cancer cell. } \\
\text { 2013;24(2):257-71 }\end{array}$ \\
\hline IL-8 FAMILY & IL-8 & CXCR1 and CXCR2 & $\begin{array}{l}\text { promote cell proliferation, angiogenesis, cancer } \\
\text { metastasis, chemoresistance, anti-anoikis, } \\
\text { maintain CCSC properties }\end{array}$ & $\uparrow$ & {$[88-94][96-99]$} \\
\hline \multirow[t]{2}{*}{ IL-10 FAMILY } & IL-10 & IL-10RA and IL-10RB & confusing & $\uparrow$ & $\begin{array}{l}\text { [123-125] Zadka L, et al. Cytokine. } \\
\text { 2018; 110: 116-25 }\end{array}$ \\
\hline & IL-22 & IL-10RB and IL-22R / IL-22BP & $\begin{array}{l}\text { promote tumorigenesis, stemness, anti-apoptosis } \\
\text { and cell proliferation }\end{array}$ & $\uparrow$ & [110-118] \\
\hline \multirow[t]{5}{*}{ IL-17 FAMILY } & IL-17a & IL17R & $\begin{array}{l}\text { promote cell cycle progression and angiogenesis, } \\
\text { facilitate the occurrence and development } \\
\text { indirectly and change the tissue environment and } \\
\text { microbiota of CRC }\end{array}$ & $\uparrow$ & $\begin{array}{l}\text { [127] Razi S, et al. Cytokine. 2019; } \\
\text { 116:7-12 }\end{array}$ \\
\hline & IL-17b & IL17R & promote tumour & $\uparrow$ & $\begin{array}{l}\text { Razi S, et al. Cytokine. 2019; } \\
\text { 116:7-12 }\end{array}$ \\
\hline & IL-17c & IL17R & null & -- & [127] \\
\hline & IL-17e & IL17R & antitumour & - & $\begin{array}{l}\text { Razi S, et al. Cytokine. 2019; } \\
\text { 116:7-12 }\end{array}$ \\
\hline & IL-17f & IL17R & $\begin{array}{l}\text { tumour suppression effect possibly by inhibiting } \\
\text { tumour angiogenesis }\end{array}$ & $\downarrow$ & $\begin{array}{l}\text { [134] Razi S, et al. Cytokine. 2019; } \\
\text { 116:7-12 }\end{array}$ \\
\hline
\end{tabular}

$\uparrow$ : upregulated, $\downarrow$ : downregulated, -: unchanged, - - confusing.

\section{IL-1 Family}

"IL-1" was first discovered in 1979 [8]. After a long period of research, it was found that the IL-1 family was comprised of IL-1a, IL-1 $\beta$, IL-1Ra, IL-18,

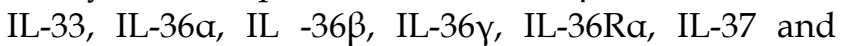
IL-38. IL-1 $\alpha$, IL-1 $\beta$, IL-1Ra and IL-33 were found to be increased significantly in CRC, while IL-18 was decreased in colon cancer (CC) patients [9-11]. IL-18Ra and IL-18R $\beta$ are IL-18 receptors, and IL-18R $\beta$ was overexpressed in rectal cancer. In mammalian CRC, ST2 was the upregulated receptor of IL-33. Furthermore, sST2, a soluble form of the IL-33 receptor, was downregulated in highly metastatic CRC cells compared with the level in low-metastatic CRC cells [10-12].

\section{Members with tumour-promoting effects}

IL-1 $\alpha$

IL-1a could negatively change the chemosensitivity, which is of great value for the clinical treatment of CRC. A recent study used HCT116 colorectal carcinoma cells as the research objects to depict the details of IL-1a-dependent protein-protein interactions (PPIs). Specifically, these cells were also used to explain the experimental phenomena showing that exogenous IL-1a with $5-\mathrm{Fu}$ changed the expression of cell adhesion molecules and that exogenous IL-1a promoted chemosensitivity in both chemosensitive and chemoresistant CRC cell lines, while endogenous IL-1a promoted chemosensitivity only in the chemosensitive HCT116 cells. The altered cell adhesion molecule suggested 
that the potential for enhanced cancer metastasis and 5-FU-induced cell death were increased with the treatment of exogenous IL-1a [13].

IL-1R1 and IL-1R2 are IL-1a receptors. Because of the pro-tumour function of IL-1a, blocking the IL-1R1 and IL-1R2 has attracted attention. Anakinra is an IL-1R antagonist. Experimental studies have shown that anakinra can reduce interstitial fluid pressure (IFP), which is measured indirectly by perfusable tissue fraction (PTF) and tumour blood flow (TBF). High levels of IFP in CRC metastasis may reduce the efficacy of antitumour drugs. The experiment showed that anakinra couldn't change the PTF or TBF, because of the experimental design and dearth of data samples, this result needs to be further studied [14]. In contrast, TRAP IL-1, which is a soluble receptor, reduced the proliferation of CRC cells by inhibiting IL-1R1 [15]. Additionally, the single immunoglobulin IL-1 receptor-related molecule (SIGIRR) downregulated IL-1R signaling, whereas a SIGIRR isoform (SIGIRR $\triangle E 8$ ) suppressed the function of SIGIRR. In mice that expressed SIGIRRN86/102S, the severity of inflammatory CRC was increased [16]. In addition, IL-1R could be used as an indicator. Patients with CRC who did not respond to CTX therapy had higher levels of IL1-R1. Moreover, for patients with consensus molecular subtype 1 (CMS1) CRC and CC subtypes 3 (CCS3), IL-1R1 can be used as a predictor of patient survival [16]. Increased IL-1R2 enhanced the expression of angiogenic factors in CC, hence, IL-1R2 may be used as a prognostic marker [17].

\section{IL-1 $\beta$}

The role of IL-1 $\beta$ in CRC is closely related to the NF-kB pathway. Through the NF-kB pathway, IL-1 $\beta$ increased the expression of miR-181a, thereby inhibiting phosphatase and tensin homologue (PTEN) expression and promoting the proliferation of CC cells [6]. In another study, IL-1 $\beta$ increased the expression of MiR301A in intestinal epithelial cells (IECs) in colitis-associated cancer (CAC) patients, thus inhibiting BTG anti-proliferation factor 1 (BTG1) expression and subsequently promoting the IL-1 $\beta$-related NF-kB pathway [18]. Therefore, blocking the NF- $\mathrm{kB}$ pathway is an idea for the future treatment of CRC. Oroxylin A [19], GEN-27 [20], tauroursodeoxycholic acid (TUDCA) [21] and an ethanol extract obtained from the aerial parts of Artemisia princeps Pampanini cv. Sajabal (EAPP) have been verified as a blocker of the NF-kB pathway, which would inhibit CRC. In addition, EAPP can inhibit tumour growth by downregulating the expression of pro-inflammatory factors including IL-1 $\beta$ and decreasing the level of anti-apoptotic proteins such as XIAP, cFLIP, survivin, and Bcl-2 [22].

The tumour microenvironment (TME) is of vital importance during the development of tumours. In CRC, the TME also affects the genesis and development of CRC, and some of these processes are related to IL-1 $\beta$. Compared with normal tissue, a high expression of IL-1 $\beta$ was connected with CRC intestinal mucosa and was also correlated with high expression of HLA class II molecules. Moreover, HLA class II triggers monocyte activation, hence increasing IL-1 $\beta$ expression [23]. In addition, LPS can trigger the expression of IL-1 $\beta$ in neutrophils. In the CAC milieu, IL-1 $\beta$ produced by neutrophils can induce intestinal mononuclear phagocytes (MPs) to produce IL-6, thereby promoting tumour formation [24]. A complement/neutrophil/ IL-1 $\beta$-myeloid cells/IL-17A axis is a recently proposed model that explains the impression of the complement system on CRC [5]. All the evidence suggests that, because of the relationship between the TME and IL-1 $\beta$, changing the TME may be a clinical strategy (Fig. 1). Resveratrol, which is a natural polyphenolic compound found mainly in grapes and their seeds, can alter the immune equilibrium between peripheral blood mononuclear cells (PBMCs) and CRC cells [25]. In addition, as a chemotherapeutic agent, CPT-11 has been used to treat a wide range of cancers, including CRC. However, its toxic effects limit its use. CPT-11 can cause neutrophil infiltration via the IL-33/ST2 axis, promote macrophage infiltration in intestinal tissues and activate the NLRP3 inflammasome, causing a surge in IL-1 $\beta$, which then leads to corresponding side symptoms [26, 27]. Fortunately, andrographolide (Andro) inhibits the NLRP3 inflammasome in macrophages in vivo and in vitro, which reduces IL-1 $\beta$ production and thereby reduces the risk of CAC [28].

\section{Members with tumour-suppressive effects}

\section{IL-18}

Inflammasome-IL-18 axis can act on natural killer (NK) cells to express fasL, thereby having a tumour-inhibiting effect [29]. Mutations in the Mediterranean fever gene (Pyrin-IL-18) axis can improve intestinal barrier integrity and prevent CRC formation [30]. The interaction of the intestinal flora with ILs has been an innovative thought for CRC treatment. A latest study shows that the commensal fungi-SYK- CARD9-IL-18 axis can prevent CC [31]. Since IL-18 can induce T cells to secrete IFN- $\gamma$, the specificity of IFN- $\gamma$ in tumours is still confusing. Furthermore, in a mammalian CRC model, a plasmid vector constructed with the human telomerase reverse transcriptase (hTERT) promoter, IL-18 and herpes simplex virus thymidine kinase (HSV-TK) had a good therapeutic effect [32]. 


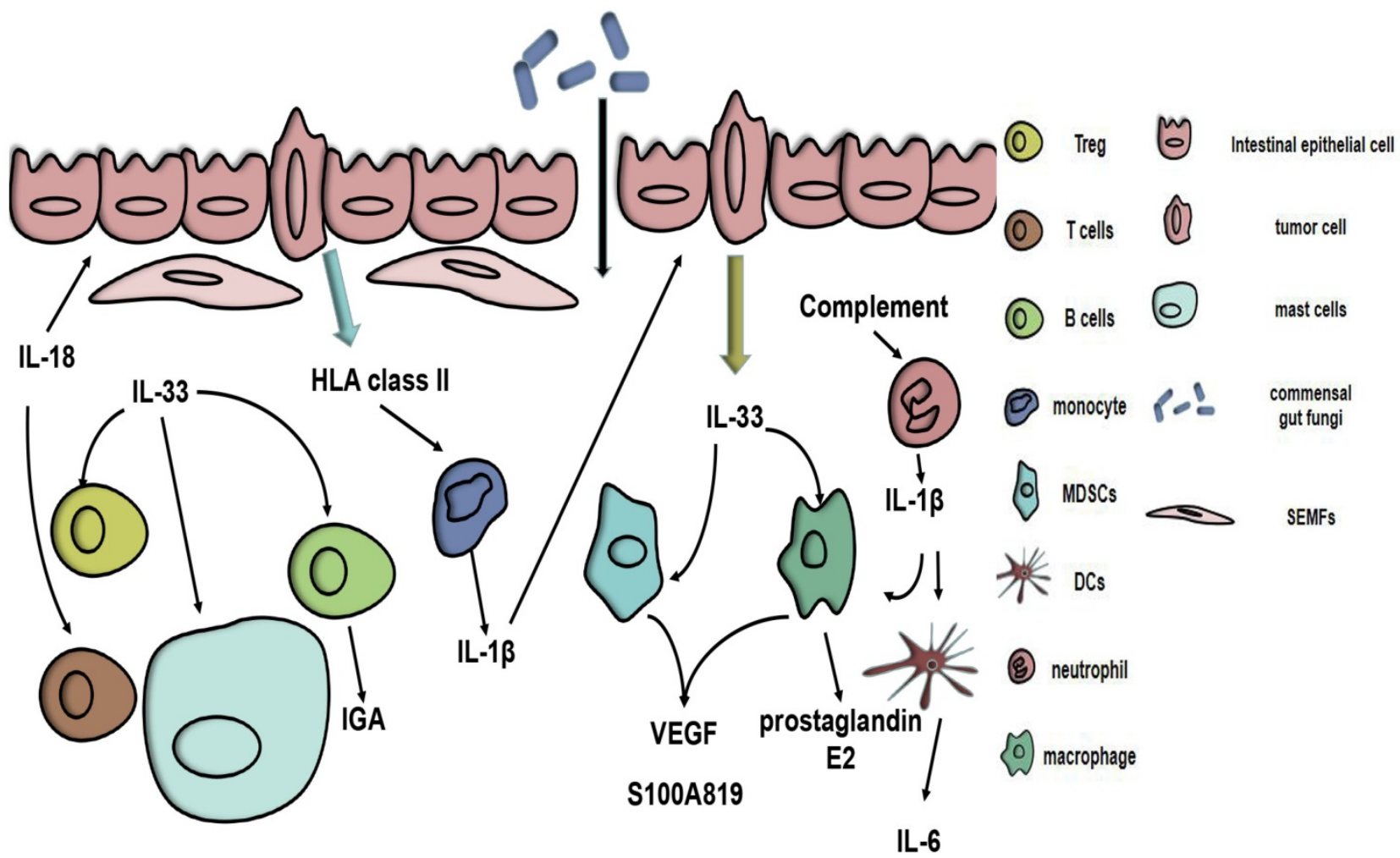

Figure 1. Potential role of the IL-1 family in colorectal cancer. HLA class II antigens expressed on CRC cells stimulate the resting monocytes to produce IL-1 $\beta$. Complement immunity triggers neutrophils to release IL-1 $\beta$, which can cause myeloid cells to produce IL- 6 and increase the IL-17A response. IL-1 $\beta$ can act on IECs and CRC cells. IL-33 can be secreted by vascular endothelial cells and tumour cells. Tumour-secreted IL-33 can act on myeloid cells, causing them to release S100A8/9 and VEGF, thereby altering the tumour microenvironment. IL-33 can also trigger macrophage production of prostaglandin E2, alter the phenotype of Tregs and activate SEMFs and mast cells. The production of IGA in B cells is triggered by IL-33. IL-18 can stimulate epithelial restitution and activate the T cell response, thus enhancing the integrity of the intestinal barrier.

\section{IL-36 \& IL-37}

The studies of IL-36 and IL-37 are limited. IL-37 was found to inhibit the development of CC cells by inhibiting $\beta$-catenin, which implies an inhibitory effect of IL-37 on CRC [33]. In human CRC, IL-36y produced by vasculature cells is associated with the conservation of the tertiary lymphoid structure (TLS). Macrophage-produced IL-36Ra is related to immunosuppressive markers, including PD-1, CTLA4 and PD-L1 immune-checkpoint markers [34]. In CRC specimens, reduced disease-free survival (DFS) and overall survival (OS) were closely related to low level of IL-37 and high numbers of CD66b+ neutrophil, suggesting that intratumoural IL-37 and CD66b+ neutrophils can be used as independent factors of clinical prognosis for CRC patients [35].

\section{Members with both tumour-promoting and tumour-suppressive effects}

\section{IL-33}

IL-33 can alter the TME by recruiting CD11b+GR1+, CD11b+F4/80+myeloid cells and macrophages $[7,36]$, activating subepithelial myofibroblasts (SEMFs) and mast cells [37], and altering the phenotype of Tregs [11], thus promoting the progression of CRC. In addition, IL-33 can affect endothelial cells to promote angiogenesis [7] and activate NANOG, NOTCH3 and OCT3/4 to enhance CC cell stemness [36]. sST2 can inhibit M2a polarization, macrophage infiltration, and Th1 and T helper 2 (Th2) responses, thereby promoting CRC [12]. A latest research found that the expression of FoxP3 mRNAs was increased in CRC tissues. IHC analysis found that sST2 was correlated with Treg cells which actively express foxp3, implying that sST2 may increase Treg cells to change the TME to make CRC more likely to develop. Additionally, sST2 was interrelated with the poor prognosis of CRC patients [38].

As ILs research progressed, it was discovered that IL-33 could suppress CRC as well. By activating $\mathrm{NF}-\mathrm{kB}$ in mesenchymal cells and inducing IFN $\mathrm{Y}$ gene expression in non-haematopoietic cells, IL-33 can suppress sporadic CC because induced inflammatory factors can exert antitumour effects [39]. In addition, in IL-33-deficient mice, the risk of CAC increased due to an increase in IL-1a, while IL-33 maintained intestinal microbiota homeostasis by triggering the production of IGA in B cells, thereby inhibiting IL-1a-induced CAC [40]. ST2 can reduce Treg infiltration and increase CD8 $+\mathrm{T}$ cells, thereby inhibiting CRC [11]. 


\section{IL-2 Family}

First described as T cell growth factor in 1976, IL-2 was found in the supernatant of mitogen-activated human $\mathrm{T}$ cells, where it played an essential role in supporting the growth and proliferation of $\mathrm{T}$ cells in vitro [41]. The basis for ensuring these biological functions is the combination of IL-2 with its receptors, which are composed of 3 subunits (IL-Ra, IL-2R $\beta$, and IL-2R $\gamma$ ). Among them, IL-2RY was found not only in IL-2, but also in IL-4, IL-7, IL-9, IL-15 and IL-21. Subsequently, these cytokines were classified into the same family, the IL-2 family (also the $\gamma$-chain family or $\gamma$ c family) [42]. As has been reported recently, IL-2, IL-9 and IL-15 were elucidated to exert antitumour effect and were regarded as promising CRC treatment candidates $[44-46,58]$. On the contrary, it was demonstrated that IL-4 and IL-7 were significantly overexpressed in CRC tissues and played negative roles in CRC progression [49-52]. Moreover, the function of IL-21 on CRC progression is controversial, with both tumourpromoting and antitumour effect have been reported [47, 48, 59-61].

\section{Members with tumour-promoting effects}

\section{IL-4}

IL-4 is produced by Th2 cells and has a wide range of effects on $\mathrm{T}$ cells, $\mathrm{B}$ cells, etc [53]. The association between IL-4 and the abnormal STAT6 activation, which mediates signal transduction and promotes metastatic processes of cancer, was recently confirmed in CRC [51]. The activation of STAT6 in its transcription is induced by the binding with specificity protein 3 (SP3) in the promoter region of STAT6 gene, which was mediated by the downstream factor of IL-4 named E2F1. Additionally, the overexpression of several epithelial-mesenchymal transition (EMT) drivers, including zinc finger E-boxbinding homeobox (Zeb) 1 and Zeb2, was also confirmed, indicating that IL-4 promoted tumour progression via E2F1/SP3/STAT6 axis [51].

Rapid tumour growth is caused mainly by abnormal cell proliferation and inhibited apoptosis, and is the basis for tumour proliferation. In recent studies, IL-4 has been reported to be highly involved in this process. IL-4 secretion regulated by the upstream miR-195/NOTCH axis was confirmed to promote rapid tumour growth through activating recruitment and polarization of the M2-like tumour-associated macrophages, which had long been considered as a risk factor for CRC progression [52]. In addition, EMT is considered to be positively related to the invasion and metastasis of tumour cells. The participation of IL-4 in EMT has been highlighted recently. As has been reported, exogenous IL-4 stimulation decreased the membranous epithelial marker E-cadherin level and increased cytoplasmic mesenchymal marker vimentin level at the mRNA and protein levels in CRC cell lines, and both E-cadherin and vimentin were biomarkers representing the stimulation of EMT in cancer cells [51]. Consistent result was also observed in another study based on the secretion of IL-4 in CRC [52].

\section{IL-7}

Different from other ILs, IL-7 is produced by non-haematopoietic stromal cells, although dendritic cells (DCs) can also produce a fraction of IL-7 [54]. IL-7 is a cytokine that enhances $\mathrm{T}$ cell proliferation and survival and has been listed as one of the "Top Agents with High Potential for Use in Treating Cancer" in 2007 [55]. Although IL-7 was detected to be elevated in CRC patients compared with the control group and the expression level was associated with metastatic disease and tumour location $[49,50]$, little progress has been made on the underlying mechanisms of IL-7-induced aggressiveness of CRC. Therefore, more advances should be made to investigate biological function of IL-7 in CRC and investigate related molecule mechanisms, which may contain the crosstalk between IL-7 and other factors in the immune system.

\section{Members with tumour-suppressive effects}

\section{IL-2 \& IL-15}

IL-2 is considered as an important cytokine that induces T-cell-mediated immune response by activating NK cells, T-cell, and is involved in the development of regulatory $\mathrm{T}$ cells [43]. Similarly, IL-15 maintains homeostasis and induces activation of NK cells and CD8+ memory $\mathrm{T}$ cells [56]. It is acknowledged that NK cells are key antitumour primary effectors to eliminate CRC cells without prior immunization, and altered phenotype and dysfunction of NK cells in CRC patients caused the limitation of the immune response and were associated with the low survival rate. Notably, a study revealed that a treatment strategy combining cetuximab, IL-2 and IL-15, stimulated NK cells and improved cytotoxicity, which provides new insights into ILs-based CRC treatment approaches [44].

IL-15 is negatively involved in CRC progression via inhibiting the proliferation and promoting apoptosis of CRC cells. In order to enhance the transfection efficiency of the gene vector that carrying IL-15, researchers developed a novel gene delivery system with a self-assembly method by MPEG-PLA and DOTAP (DMA), denoted as DMA-pIL15, and the transfected CRC cells showed effective high level of 
IL-15 secretion [46]. Moreover, Ki67 staining and TUNEL assay were applied to determine the regulatory role of IL-15 on cell proliferation and apoptosis, and the results showed that lower proliferation rate and higher TUNEL-positive rate (representing a higher apoptosis rate) were shown in DMA-pIL15-treated group in comparison with the control group, which demonstrated that IL-15 exhibited antitumour effect via inhibiting the proliferation and promoting apoptosis of CRC cells. Also of note, for most malignant solid tumours, the formation of large numbers of microvessels constitutes the basis for tumour growth and metastasis. By counting the number of CD31-positive vessels in the field, the overexpression of IL-15 caused by gene vector was confirmed to reduce angiogenesis of CRC, which further suggested the positive effect of IL-15 in the invasion and metastasis of CRC cells [46].

\section{IL-9}

Produced by Th2 cells, Th9 cells, Th17 cells and regulatory T cells, IL-9 is a regulatory cytokine and is known for promoting the proliferation and growth of mast cell [57]. In CRC, it was demonstrated that IL-9 was predominantly produced by bona fide Th9 cells. By binding to IL-9R expressed on CD8+ T cells, IL-9 significantly enhanced the expansion of its targeted cells, thus inhibiting CRC progression. Additionally, the biological effect of IL-9 on the immune response was significantly impaired by PD-1/PD-L1-mediated inhibition, which was regarded as a key oncogenic signaling pathway [45]. A recent study also confirmed antitumour effect of IL-9, followed by the evidence that the expression levels of IL-9 and its mRNA in CC tissue specimens are significantly lower than those in adjacent tissues [58]. This experimental team also demonstrated that IL-9 overexpression inhibited tumour growth in vivo and showed that this effect was strengthened by activating regulatory $\mathrm{T}$ cell to have the killing effect on CC cells [58].

\section{Members with both tumour-promoting and tumour-suppressive effects}

\section{IL-2 1}

IL-21 is produced by NK cells, CD4+ T cells and TH17 cells, and can act on all lymphocyte subsets, DCs and smaller monocytes to enhance the intensity of the immune response [48]. It was confusing that both pro-tumour and anti-tumour effect of IL-21 on CRC have been reported. As one of the Th17-derived cytosines, IL-21 was broadly reported to be an essential proinflammatory mediator and promoted CRC progression [59-61]. Additionally, the elevated expression level of IL-21 was detected in CRC microenvironment, and it was revealed that IL-21 level was negatively correlated with poor survival rate, suggesting its potential role as a prognostic biomarker [47]. On the contrary, IL-21 was also reported to exhibit antitumour effects. CD4+CXR5+PD-1-follicular helper $\mathrm{T}$ cell was confirmed to facilitate the expansion of CD8+ T cells via the secretion of IL-21, thus enhancing the expression of CD107a and IFN-y [48]. However, similar to IL-9, the antitumour effect could also be suppressed by the activation of PD-1/PD-L1 signaling pathway [48]. In a word, further investigations are urgently needed to figure out the regulatory role of IL-21 in CRC as well as the underlying mechanisms.

\section{IL-6 Family}

The IL-6 family comprises IL-6, IL-11, IL-27, IL-31, leukaemia inhibitory factor (LIF), oncostatin M (OSM), ciliary neurotrophic factor (CNTF), cardiotrophin-like cytokine factor 1 (CLCF) and cardiotrophin 1 (CT-1), which all have the similar structure [62-64]. The IL-6 family has recently received widespread attention because of its emerging role in various diseases, such as infection, chronic inflammation, autoimmunity and cancer. Initially named B-cell stimulatory factor 2 (BSF-2), IL-6 was officially given the name IL-6 by The New York Academy of Sciences in 1988 [65]. IL-6 is mainly produced by macrophages, as well as bone marrow-derived myofibroblasts (BMFs), DCs, IECs and myeloid cells [66-69].

\section{Members with tumour-promoting effects}

\section{IL-6}

Researches show that IL-6 regulates the progression of CRC when combined with gp130 mainly in three signaling pathways, Shp2-Ras-ERK, JAK1/2-STAT3 and PI3K-Akt-mTOR. The factor in common for most of these pathways is STAT3, which plays a dominant role in all of these pathways [66]. A recent study also proved that STAT3 activated by IL-6 played a critical role in the fibroblast activation [70]. In a latest study on Wu Mei Wan (WMW), which is a traditional Chinese medicine published in Treatise on Febrile Disease, NF-кB/IL6-STAT3 signaling pathway plays an important role. By making AOM/DSSinduced CAC mouse model, WMW shows a great curative effect on CAC. In this study, it is of great focus on NF-kB/IL-6/STAT3 pathway when exploring the tumour suppression mechanism of WMW [71].

In the progression of $\mathrm{CRC}$, the regulation of each stage of the tumour can be accomplished through these molecular pathways. For example, a study proved that the NF-KB-IL6-STAT3 pathway promoted CRC [72]. The activation of the IL-6/STAT3/ERK 
signaling pathway facilitates the angiogenesis, migration and proliferation of CRC. By activating the JAK2/STAT3 pathway, IL-6 induces the EMT in CRC cells through the $\beta$-catenin/Wnt signaling pathway [73]. The IL-6R/STAT3/MIR34A feedback loop is also necessary for the EMT and metastasis of CRC cells [74]. Through the STAT3 pathway, exogenous IL-6 induces the secretion of tumour-derived IL- 6 to create a microenvironment that is favourable for the metastasis of CRC cells [75]. Another study found that IL-6 contributed to the environment described above by controlling the secretion of mucin [74]. In an up-to-date study, IL-6 has been shown to be involved in the drug resistance of CRC under hypoxia [76]. The relationship between IL-6 and other cytokines has been extensively studied. For example, IL-6 enhances tumour angiogenesis in CC through the expression of VEGF [77]. At the genetic level, pleiotropic IL-6 secreted by senescent cells has the effect of promoting mitosis [78], and through the STAT3/Gp130 pathway, IL-6 promotes the expression of downstream genes covered by cyclin D1, c-myc, bcl-XL, survivin, etc. [79].

\section{IL-11}

Similar to IL-6R, IL-11 receptor is a member of the gp-130 dependent receptor group [80]. IL-11 plays its part in CRC principally by the similar pathways, like JAK/STAT signaling pathway [81]. A previous study shows that IL-11 can promote the migration and proliferation of CRC cells by activating PI3K and P44/P42 MAPK pathways [82]. In recent years, there have been few studies concentrate on the effect of IL-11 on CRC. In recent studies, IL-11 prefers to appear in the gp130-STAT3 pathway together with IL-6 as a drug or clinical therapy target. For example, a latest study shows that Bazedoxifene can suppress the effect of IL-11 by inhibiting the phosphorylation of STAT3 and nuclear translocation in CRC [79].

In summary, researchers are more interested in IL-6 than IL-11. IL-6 promotes the growth, angiogenesis, proliferation, migration and formation of the microenvironment on CRC through different pathways. Consequently, the level of IL-6 is also involved in the cytokine profile to show the progress and treatment effect on CC [83]. Recent studies on IL-6 have mainly focused on the EMT, metastasis and TME, which contributes to the treatment of CRC. For example, nonsteroidal anti-inflammatory drugs and metformin were used to inhibit IL-6-mediated EMT in CRC, showing a good result in CRC regression [84-86]. As an interleukin which has been thoroughly researched in a long time, IL-6 deserves more attention to drug tests and clinical treatment. As cytokines which have similar receptors and pathways,
IL-11 may deserve more separate researches to find more inspiration on the treatment of CRC.

\section{IL-8 Chemokine Family}

IL-8 (CXCL8), as a member of the CXC family, is mainly induced by pro-inflammatory cytokines such as IL-1 $\beta$ and TNFa to recruit and activate neutrophils and granulocytes to the inflammation place. IL- 8 has an affinity for two types of receptors: CXCR1 and CXCR2. Ligation of IL-8 with different receptors causes different biological outcomes.

\section{Members with tumour-promoting effects}

\section{IL-8}

Three signaling pathways have been described for IL-8: a major pathway through the activation of intracellular signaling PI3K that induces phosphorylation of its substrate, Akt, which plays an essential role in regulating cell survival, migration and angiogenesis. Other pathways include the MAPK signaling cascade and migration- associated PLC-dependent PKC signaling pathway. In addition, activation of the non-receptor tyrosine kinases Src and FAK contributes to IL-8-mediated cancer cell proliferation, survival and chemoresistance, and Rho-GTPases are also involved in IL-8-induced cancer cell mobility and invasion [87]. In recent years, researchers have gradually revealed the exact function of IL-8 against CRC, showing the great progress made in the treatment of CRC patients.

IL-8 is a significant chemotactic stimulus that influences the growth and invasion of CRC cells through different mechanisms. High levels of IL-8 in CRC tissue are correlated with higher tumour grade and increased invasion into the liver. In addition, the level of IL-8 also benefits CRC diagnosis and high level of IL-8 in serum contributes to CRC growth and progression, indicating as a potential biomarker for CRC prognosis [88-90]. IL-8 participates in most phases of tumour development from cell proliferation and angiogenesis to cancer metastasis, moreover, some evidence has shown that IL-8 is associated with chemotherapeutic responses [91-93].

IL-8 is involved in CRC growth. Ligation of IL-8 to CXCR1 leads to CRC cell proliferation and angiogenesis. In a CRC murine model inoculated with CRC stem cell (CCSC) in vitro, cancer cells deficient in IL-8 or CXCR1 showed decreased proliferation and angiogenesis in CCSC. Recent evidence shows the dysregulated expression of cell-cycle proteins, with a reduction in cyclin D and cyclin $B$ and upregulation of the CDK inhibitory protein P21, was the underlying mechanism for IL-8 effects [94]. Similarly, in another study, incubation with a CXCR2 neutralizing antibody in HCT116 colorectal carcinoma cells 
inhibited cell proliferation. Moreover, in this study, the HCT116 cells lost chemoresistance to 5fluorouracil (5-FU) [91]. 5-FU is a major CRC drug that has shown poor outcomes in patients in recent years due to chemotherapeutic resistance. Further investigations found that IL-8 mediated the chemoresistance due to modulating multidrug resistant 1 (MDR1) via IKK- $\beta /$ p65 signaling within CRC cells treated with doxorubicin (another clinical drug for CRC) [93]. In a CRC cell model induced by HT-29 CRC cell conditioned medium, IL-8 was highly elevated and the conditioned medium induced angiogenesis was attenuated after IL-8 was neutralized, indicating that IL-8 plays a role in CRC angiogenesis. Notably, researchers found that the expression of IL-8 was decreased when curcumin or (-)-epigallocatechin-3-gallate were added, both of which are natural components found in plants [95].

Additionally, IL-8 was also found to play a central role in CRC metastasis by inducing the EMT or resistance to anoikis. A protein-protein network analysis demonstrated that IL- 8 was one of the four hub genes associated with CRC metastasis [96]. Further investigations showed that IL-8 was significantly higher in patients with stage $\mathrm{T} 3$ or $\mathrm{T} 4$ CRC, lymph node metastasis or liver metastasis. Treatment of the colorectal carcinoma cell line SW480 with IL-8 and IL-20 combined was shown to induce the expression of an EMT phenotype, by triggering the PI3K/AKT-ERK1/2 cross-talk signaling pathway, while treatments with IL-8 or IL-20 alone induced only an EMT-like phenotype, which suggested that IL-8 influenced but did not drive CRC metastasis [97]. In another study, IL-8 was correlated with anoikis resistance by ERK and AKT activation and TOPK upregulation, which consequently enhanced CRC metastasis. The addition of IL-8 to CRC cells decreased the apoptosis rate and increased Bcl-2 expression, which is an important apoptosis suppressor factor. Interestingly, IL-8 only decreased apoptosis when the cells cultured in suspension lost cell-cell adhesion yet not under cell attachment conditions. In the same case, further investigation demonstrated that the IL-8 downstream pathways of PI3K/AKT and ERK were involved in an anti-anoikis effect. In addition, elevated TOPK expression under IL-8 treatment was shown to be inhibited by the AKT inhibitor MK2206, suggesting that TOPK may be the downstream signaling target of AKT during anti-anoikis action [89]. The elevated level of IL-8 was shown to upregulate integrin av $\beta 6$ in a dose-dependent manner, which consequently contributed to liver metastasis via the ERK and Ets-1 signaling pathways. Integrin av $\beta 6$ plays a vital role in the proliferation, apoptosis, metastasis and matrix metalloproteinase secretion of CRC, and human CRC cells with silenced av $\beta 6$ show a reduction in IL-8-induced migration [98]. Besides, recent studies reported that IL-8 was required for the expression of cancer stem cell (CSC) properties through protein O-GlcNAcylation promotion, glucose uptake stimulation and glucose transporter 3 (GLUT3) and glucosamine fructose-6-phosphate aminotransferase (GFAT) upregulation [99].

Studies have also revealed some upstream regulators of IL-8. Several regulators induce IL-8 production, such as SRSF3-TRs, NTPDase2, human neutrophil peptides (HNPs), ring finger domain protein 183 (RNF183) and circulating cell-free DNA (cfDNA), each of which triggers IL-8 expression in terms of signal transduction, activation of intracellular signaling pathways and regulation of gene expression [100-104]. For instance, human neutrophil peptides (HNPs) induced IL-8 expression upon the binding of the P2 receptor or P2Y6 and the phosphorylation of components in the ERK1/2 signaling pathway [100]. Adenylate kinase (ADK) sustainably activated the P2 receptor signaling pathway, and cfDNA activated TLR9-MyD88 signaling, thereby inducing IL-8 expression [101, 102]. Studies also found some regulators that inhibited IL-8 expression, including microRNA-204 (miR-204) and DUSP2 [105,106]. DUSP2 regulates IL-8 via ERK1/2 signaling and the $\mathrm{C} / \mathrm{EBPa}$ transcriptional factor. Hypoxia inhibits the expression of DUSP2, leading to the elevation of IL-8, which indicates that the hypoxia-dusp2-IL-8 pathway may be considered a new therapeutic approach [106] (Fig. 2).

Taken together, IL-8 absolutely is a critical cytokine for CRC progression. Researches of IL-8 on CRC metastasis are relatively more mature than other function investigations. According to studies mentioned above, immunotherapy combining IL-8 and IL-20 may be a nice strategy for inhibiting CRC metastasis. On the other hand, it requires further researches to investigate detailed molecular mechanisms of IL-8 in enhancing angiogenesis and maintaining CSC properties. Surprisingly, the curcumin or (-)-epigallocatechin-3-gallate for decreasing the level of IL-8 in CRC has brightened our eyes, which has potential for developing as a new chemotherapy drug. Though, more researches are required to unveil the underlying mechanisms.

\section{IL-10 Family}

Six direct members are involved in IL-10 family: IL-10, IL-20 subfamily members IL-19, IL-20, IL-22, IL-24, IL-26, and a distant family group of IFNs, containing IL-28A, IL-28B and IL-29 [107]. Studies have shown that two family members (IL-10 and IL- 
22) are closely related to $C R C$, suggesting that they are potential therapeutic prospects. IL-10 was first discovered to be secreted by Th2 cells in mice in 1989, and since it inhibited the synthesis of IL-2 and IFN- $\gamma$, it was primarily represented as secreted cytokine synthesis inhibitory factor (CSIF). IL-22 is another essential cytokine in CRC. First discovered as IL10-related T cell-derived inducible factor (IL-TIF), it can be produced by most of the lymphocyte subsets, which mainly are ILC3, Th17 and Th22 [107-110].

\section{Members with tumour-promoting effects}

\section{IL-22}

IL-22 exerts its biological effects by binding to its heterodimer receptor complex which consists of two subunits, IL-10R2-specific and IL-22-specific receptor IL-22. In addition to its functional receptor, an endogenous antagonist, IL-22-binding protein (IL22BP, known as IL-22RA2) is a natural agent to inhibit IL-22 bioactivity [111]. The primary downstream signaling targets of IL-22 are STAT3, STAT1 and STAT5, which are activated by the phosphorylation of Jak1 and Tyk2. Moreover, IL-22 also induces the activation of the MAPK, NF-kB and PI3K-Akt-mTOR signaling pathways.
As IL-22 influences gut homeostasis, it is intimately involved in CRC. In colorectal tissue, IL-22 is a significant tumour-promoting cytokine influencing tumorigenesis, stemness, anti-apoptosis and cell proliferation. High levels of Th22 and IL-22RA1 in a colorectal site are associated with CRC. The increase in Th22 represents a shift from early stage tumour progression to the advanced stage, and a reduction in IL-22RA1 gene expression correlates with low differentiated CRC grade and worse patient outcomes [112,113].

The role of IL-22 in the process of carcinogenesis may be attributed to DNA damage induced by synergism with IFN-ץ or helicobacter hepaticus $(\mathrm{Hh})$ infection to produce nitrogen oxide intermediates (iNOS). As reported previously, in a RAG-deficient murine model of carcinogenesis induced by $\mathrm{Hh}$, the iNOS and NO expression was very low in IL-22-deficient mice. However, tumour development was more rapid, and the degree of DNA damage was higher in the control group. Another study showed that IL-22 potently synergized with IFN- $\gamma$ for iNOS expression via STAT3 activation in human DLD-1 colon carcinoma cells $[114,115]$.

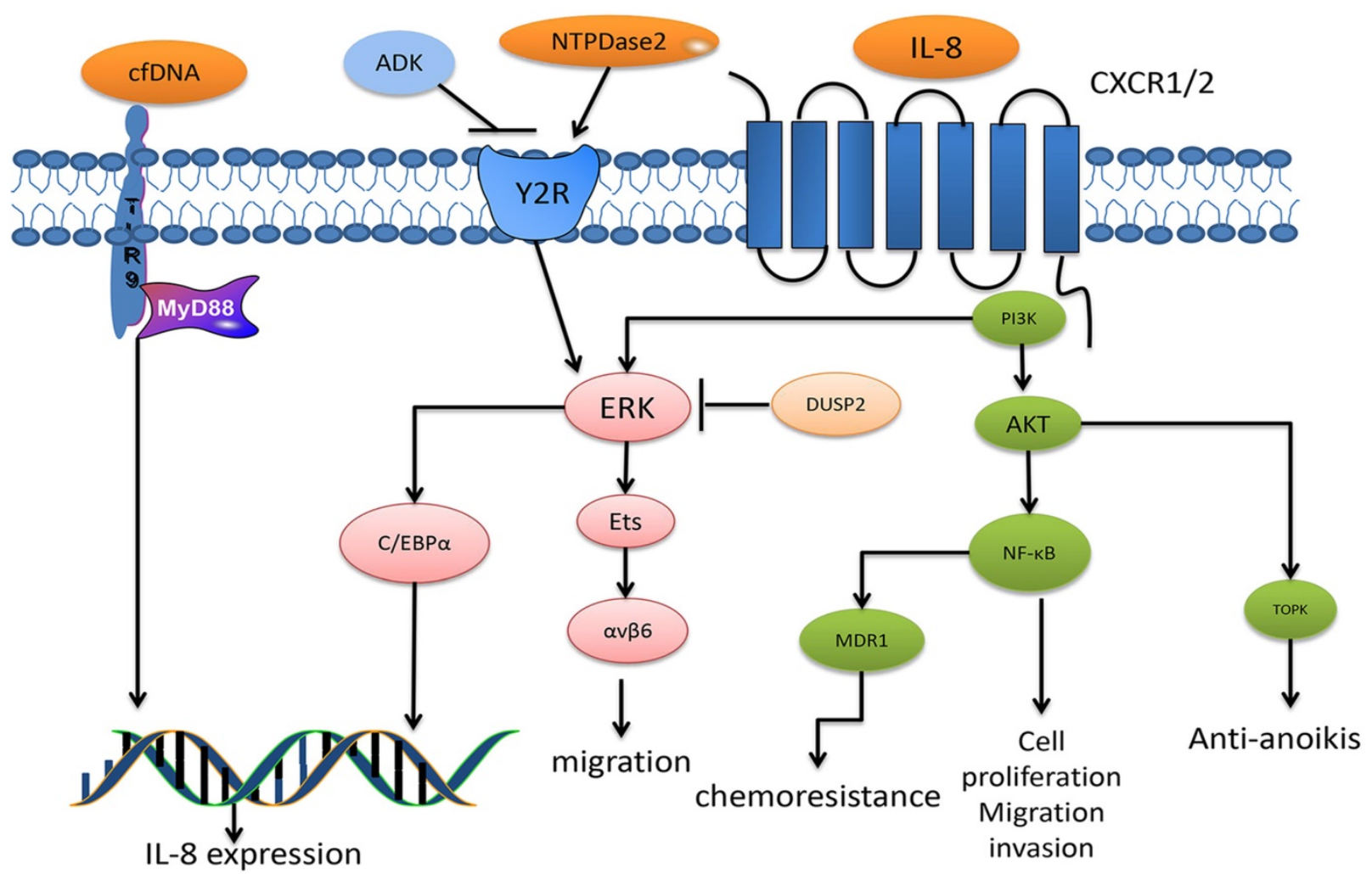

Figure 2. The diagram summarizes the major signaling pathways of IL-8 in CRC. IL-8 binds to G protein-coupled receptor CXCR1 or CXCR2, leading to the activation of PI3K, which induces the phosphorylation of Akt and ERK, respectively. The Akt signals have been reported to activate transcription factors NF-KB, which is associated with CRC cell proliferation, migration and invasion, and is particularly correlated with chemoresistance after MDR 1 activation. In addition, TOPK, reported a downstream factor of Akt, mediates anti-anoikis. Another ERK induces the phosphorylation of Ets transcription factors, thus upregulating integrin aßv6 expression, which mediates CRC migration. In addition, ERK signaling also activates C/EBPa, thus promoting IL-8 expression. DUSP2 is a termination factor of ERK activation, and studies have reported its inhibitory role against ERK in CRC. Cell-free DNA activates TLR9-MyD88 signaling, thus promoting IL-8 expression in CRC. NTPase2 binds to Y2R to activate ERK signaling, especially that which leads to mediated IL-8 expression. In addition, the ADK antagonist NTPase2 functions by binding to Y2R. 


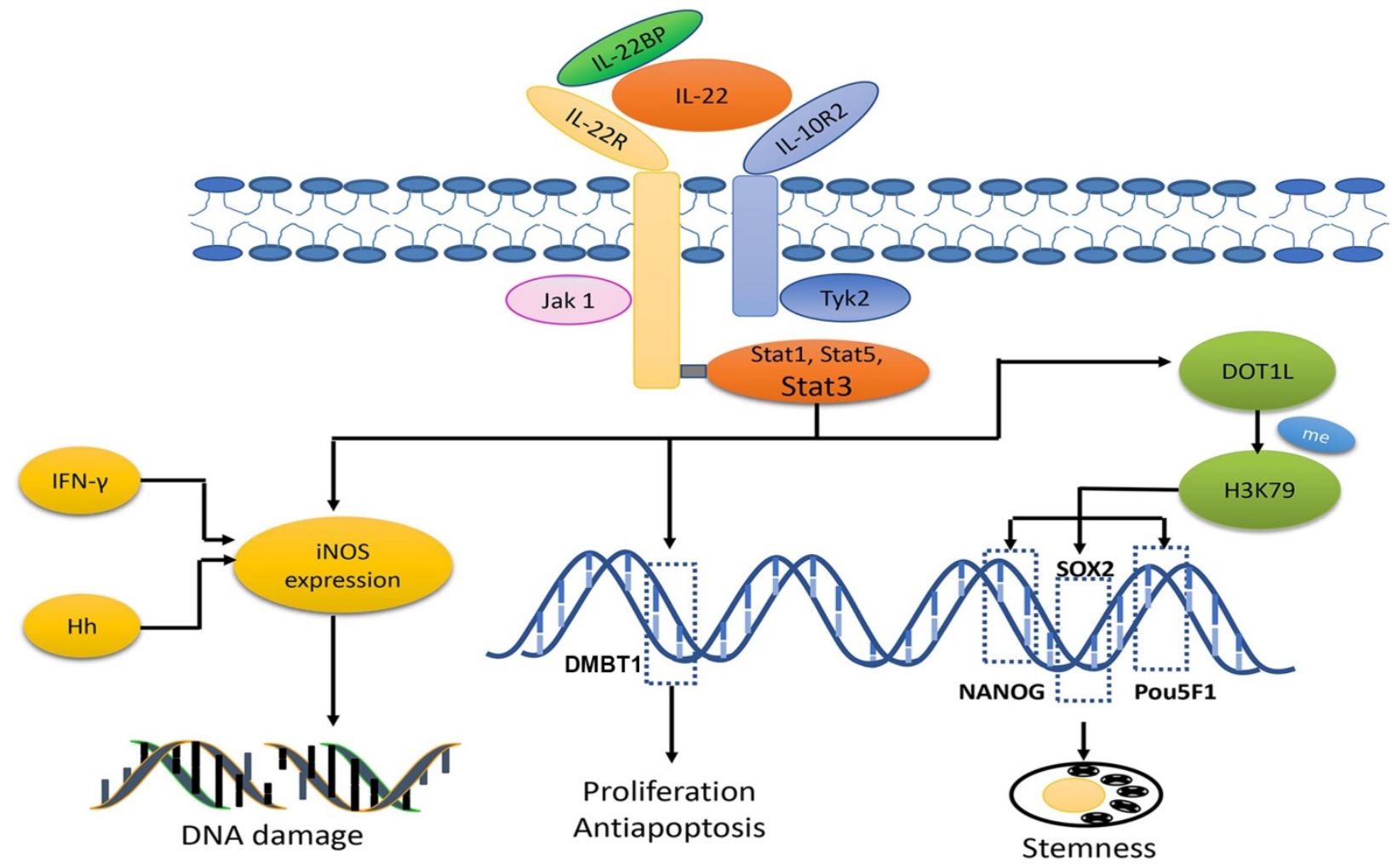

Figure 3. IL-22 signaling is transduced through heterodimer receptor complexes composed of IL-10R2 and IL-22. In addition, there is an endogenous antagonist, IL-22BP. The primary downstream signaling targets of IL-22 are STAT3, STAT1 and STAT5, which are activated by the phosphorylation of Jak1 and Tyk2. The role of IL-22 in the process of tumorigenesis is attributed to DNA damage induction through its synergistic interaction with IFN- $y$ or Hh infection to produce nitrogen oxide intermediates (iNOS). Furthermore, STAT3 can bind to the DMBT1 promoter region, thereby promoting tumour progression. In addition, IL-22 mediates tumour stemness, which is associated with methylation of $\mathrm{H} 3 \mathrm{~K} 79$ at three core cancer stemness genes, NANOG, SOX2 and Pou5F1.

IL-22 is also involved in CRC progression. It is involved in cell proliferation, differentiation, apoptosis and invasion through STAT3, NF-kB, ERK1/2 and so on $[116,117]$. Among them, STAT3 plays a dominant role. Treated with IL-22, RKO colorectal carcinoma cells showed enhanced cell proliferation and reduction in apoptosis, whereas these effects could be eliminated after the addition of STAT3 inhibitor S3I-201 [118]. Further studies indicated that STAT3 can bind to the DMBT1 promoter region, thereby promoting tumour progression. DMBT1 was originally identified as a tumour suppressor gene in medulloblastoma, whereas accumulating evidence confirms that DMBT1 produces effects in innate immunity and epithelial cell differentiation and binds to viral or bacterial pathogens. In this study, DMBT1 was shown to be upregulated in SW403 CRC cells treated with IL-22 [116]. These studies indicate that IL-22 promotes the proliferation and inhibits apoptosis of cancer cells via the STAT3 signaling pathway.

IL-22 has been regarded as a regulator of CCSC self-renewal and expansion. Targeting IL-22 activation in CCSC-induced CC with IL-22 antibody dramatically reduced primarily tumour volume, delayed tumour development and increased mouse survival. The results inspired the authors to investigate the mechanisms undergirding these findings, and they discovered that IL-22-mediated tumour stemness was associated with the methylation of H3K79 in three core cancer stemness genes: NANOG, SOX2 and Pou5F1. Their investigation also proved that p-STAT3 can bind to the disruptor of the telomeric silencing 1-like (DOT1L) promoter region and thus induce DOT1L overexpression, which plays a key role in the methylation of H3K79 [119].

Previous studies reported that IL-22BP played a crucial role in controlling the expression of IL-22. Infected with an IL-22BP overexpression vector in C26 CC cells, the effects of tumour angiogenesis and anti-apoptosis were attenuated, and the expression of p-STAT3, VEGF-A, Bcl-xL and survirin was downregulated, which provided a theoretical basis for the use of IL-22BP in the treatment of CRC patients [120].

In summary, IL-22 has dominant performance in CRC carcinogenesis and stemness, pointing that IL-22 identified as an early stage tumour target could be a proper thinking. Though IL-22 relevant cells and receptors are represented as biomarkers for CRC prognosis and diagnosis, there's no direct evidence to confirm that IL-22 can be used as a biomarker. IL-22 $\mathrm{BP}$ is a natural factor for attenuating IL-22 effects in tumour angiogenesis and anti-apoptosis, which 
showing a prospective potential for CRC treatment (Fig. 3).

\section{Members with both tumour-promoting and tumour-suppressive effects}

\section{IL-10}

With a high binding affinity for IL-10RA and IL-10RB heterodimer [121, 122], IL-10 conducts to the recruitment and activation of JAK to initiate the downstream signaling pathway and transcription factors (STAT3, STAT1 and STAT5). Among them, STAT3 plays a dominant role in IL-10 signaling.

IL-10 is a crucial immunosuppression agent, and the lack of IL-10R in colorectal tissue could cause severe spontaneous colitis, which poses a risk for CRC initiation [123]. However, the role of IL-10 in cancer pathogenesis and development is complex. In combination with $\mathrm{CY}$, lentivectors encoding shRNA specific to IL-10 (shIL-10 LVs) silenced IL-10 expression and inhibited CRC growth. Moreover, the author discovered that IL-10 deficiency enhanced the efficacy of DC-based immunotherapy, reduced MDSC and Treg levels in the TME and promoted Th1-type antitumour responses, indicating that IL-10 plays a tumour-promoting role in CRC [124]. However, in another study, a murine tumour model treated with genetically modified lactic acid bacteria (GM-LAB) and engineered to produce IL-10 or antioxidant enzymes showed CC tumour inhibition [125], indicating that IL-10 might suppress tumour growth. The expression level of IL-10 was found lower in patients 7 days after CRC surgery than before, and patients with recurrence CRC after the surgery had significantly higher level of IL-10, indicating that IL-10 can be a prognostic biomarker in CRC [126]. Anyway, whether IL-10 is a tumour promoting agent or inhibitor still needs further studies to elucidate.

\section{IL-17 Family}

The IL-17 family consists of 6 cytokines which are structurally related, IL-17A (also called IL-17), IL-17B, IL-17C, IL-17D, IL-17E (also called IL-25) and IL-17F [127]. These family members perform distinct functions. For example, IL-17F is involved in mucosal host defence; IL-17E was confirmed to be an amplifier of TH2 immune responses, and IL-17A shows the highest involvement in tumour progression (including CRC), as well as inflammation and autoimmunity [128]. In previous studies on these cytokines, IL-17A and IL-17F catch our eyes. While IL-17A increased tumour growth and metastasis, the IL-17F has anti-tumour effects on CRC [61].
Members with tumour-promoting effects

IL-17A

IL-17A is involved in the growth, angiogenesis and metastasis of CRC. IL-17A indirectly promotes the pathogenesis and development of CRC by inducing the secretion of IL-6 through the STAT3 pathway [129]. Additionally, IL-17A increases the levels of cytokines and chemokines produced by myeloid cells to change the tissue environment and microbiota in CRC [130]. A previous study found that IL-17A in CRC cells increased the expression of Sca-1 [131], which facilitated cell cycle progression and promoted the tumorigenesis of CRC. Through IL-17-STEAP4-XIAP axis, CC can be promoted by inducing copper uptake in the inflammatory response [132]. Moreover, by binding to its receptor on vascular endothelial cells, IL-17A promotes the secretion of VEGF, thus inducing tumour angiogenesis [133] (Fig. $4)$.

\section{Members with tumour-suppressive effects}

IL-17F

In a previous study, we found that compared with the expression in normal human colon epithelial cell, IL-17F was observably decreased in CC tissue. In this study, IL-17F was also proved to have the tumour suppression effect in CC possibly by inhibiting tumour angiogenesis [134].

In summary, we speculate that IL-17 has great potential for the treatment of CRC and a study shows that the overexpression of IL-17A may be closely related to the poor prognosis in patients with CRC [135]. Besides, the underlying molecule mechanisms on IL-17A and basic function on IL-17F remain to be further studied before more effective clinical therapies and drugs are found.

\section{Clinical Application}

Since the role of ILs in CRC has been extensively studied in the laboratory and the prominent roles of ILs in CRC have been found, the clinical application of ILs in CRC treatment has attracted attention (Table 2).

\section{Playing the role directly}

Injecting ILs with a tumour suppressing effect in CRC patients is a straight and effective manner. A daily dose of pegylated recombinant human IL-10 (AM0010) was injected subcutaneously into the recruited patients with advanced solid tumours, including CRC, renal cell cancer (RCC), etc. Although there was a certain degree of side effects, such as anaemia, fatigue, thrombocytopaenia, fever, and injection site reactions, the side effects remained within an acceptable range. In this phase I study, 
according to immune-related response criteria, it was confirmed that AM0010 had a good antitumour effect on many solid tumours, including CRC, indicating that it is worthy of more clinical trials in the future [136].

\section{Targeting ILs in CRC treatment}

To improve CRC outcomes in clinical trials, inhibiting ILs that promote CRC became the choice treatment in clinical trials. MABp1, derived based on the human immune response, is a monoclonal antibody against IL-1a. In a phase III trial, the researchers proposed a new and useful evaluation standard: the primary endpoint, which refers to a stable increase in lean body mass (LBM) and stabilization or improvement in at least two signs as determined by the EORTC QLQ-C30 (fatigue, pain, and anorexia). Among those recruited with metastatic CRC, 33\% of the patients who received at least one dose of MABp1 and 19\% of the patients who received at least one dose of placebo achieved the primary endpoint $(p=0.0045)$. The two groups have shown no significant differences in serious adverse events. This finding indicated that MABp1 provided a new strategy for the treatment of advanced CRC [137]. HuMax-IL8 (now known as BMS-986253) is a monoclonal antibody with the ability to inhibit IL-8. After receiving HuMax-IL8 treatment, 15 patients with solid tumours, including 4 CRC patients, were safe without severe side effects. At the same time, their serum was tested and a decrease in IL-8 was found. This is the first trial on IL- 8 blockade, and clinical trials on BMS-986253 are continuing [138]. However, the outcome was different for another drug, siltuximab, which is a monoclonal antibody with a high affinity for IL-6. In a Phase I/II clinical trial, siltuximab had no effect on solid tumours, including Kirsten rat sarcoma-2 (KRAS)-mutant cancers [139]. Nevertheless, the findings from this experiment indicated a decrease in p-STAT3 after siltuximab treatment, and the researchers speculated that siltuximab had no clinical effect possibly because of tumour autocrine IL-6 and tumour heterogeneity.

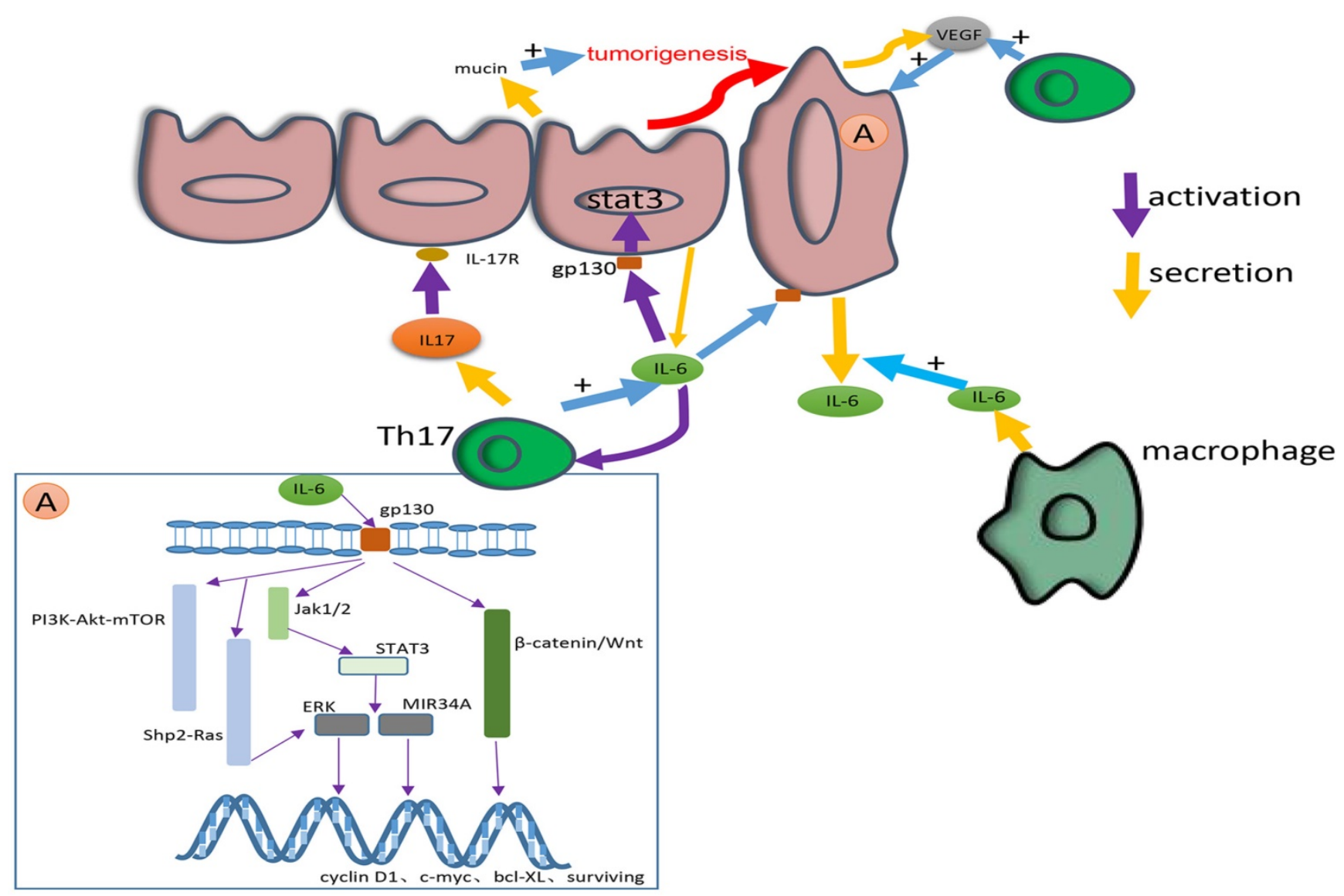

Figure 4. This picture shows the secretion, signaling pathways and cross-talk of IL-17 and IL-6. IL-6 is mainly secreted by macrophages in epithelium and colorectal cancer tissue. IL-17 is mainly secreted by Th17. IL-17 can promote the secretion of IL- 6 . In addition, IL- 6 can activate Th17. The IL- 6 secreted by macrophages can induce the secretion of tumour-derived IL-6. IL- 6 exerts its effect by combining with gP130 to activate various pathways, including the IL-6/STAT3/ERK, JAK1/2-STAT3, $\beta$-catenin/Wnt, PI3K-Akt-mTOR and Shp2-Ras-ERK pathways. Through these pathways IL-6 can change the expression of tumour cell genes such as cyclin D1, c-myc, bcl-XL, survivin, etc., to great effect. IL-6 can also facilitate the secretion of mucin and promote the tumorigenesis. IL-17 consisting of IL-17R facilitates the secretion of VEGF and promotes tumorigenesis and angiogenesis. Both IL-6 and IL-17 contribute to a microenvironment that is favourable for the growth of colorectal cancer. 
Table 2. Clinical trials targeting interleukins in CRC

\begin{tabular}{|c|c|c|c|c|}
\hline Clinical trial.gov identifier & Targeted molecule & Phases & Status & Type of CRC \\
\hline NCT01902849 & IL-6 IL-10 & $\mathrm{N} / \mathrm{A}$ & Completed & Colorectal Cancer \\
\hline NCT00072098 & IL-12 & Phase 1 & Terminated & Colorectal Cancer | Metastatic Cancer \\
\hline NCT00003046 & IL-12 & Phase 1 & Completed & Stage IV Colorectal Cancer \\
\hline NCT03542799 & IL-12 & Phase 1 & Not yet recruiting & Metastatic Colorectal Cancer \\
\hline NCT00004074 & IL-12 & Phase 1 & Completed & $\begin{array}{l}\text { Metastatic Colorectal Adenocarcinoma | Recurrent Colorectal } \\
\text { Carcinoma | Refractory Colorectal Carcinoma | Stage IV Colorectal } \\
\text { Cancer }\end{array}$ \\
\hline NCT00020267 & IL-2 & Phase 1 & Completed & Colorectal Cancer \\
\hline NCT01300858 & IL-12 & Phase 1| Phase 2 & Terminated & Metastatic Colorectal Cancer \\
\hline NCT00019591 & IL-2 & Phase 1| Phase 2 & Completed & Colorectal Cancer \\
\hline NCT00019591 & IL-2 & Phase 1| Phase 2 & Completed & CRC \\
\hline NCT00841191 & IL-6 & Phase 1| Phase 2 & Completed & Metastatic Colorectal Cancer \\
\hline NCT00841191 & IL-6 & Phase 1| Phase 2 & Completed & CRC \\
\hline ChiCTR1900023583 & IL-1 & Phase 2 & Not yet recruiting & Colorectal Cancer \\
\hline NCT03823079 & IL-11 & Phase 2 & Not yet recruiting & Recurrent Colorectal Carcinoma \\
\hline NCT03823079 & IL-11 & Phase 2 & Completed & $\begin{array}{l}\text { Recurrent Colon Cancer | Stage III Colon Cancer | Stage IV Colon } \\
\text { Cancer }\end{array}$ \\
\hline CTR20191045 & IL-1R & Phase 2 & In the recruitment & Metastasis of Colorectal Cancer \\
\hline NCT02090101 & $\mathrm{IL}-1 \beta$ & Phase 2 & Completed & CRC \\
\hline NCT02919644 & IL-2 & Phase 2 & Active, not recruiting & Metastatic Colorectal Cancer \\
\hline NCT03222089 & IL-2 & Phase 2 & Withdrawn & Colorectal Cancer \\
\hline NCT03610490 & IL-2 & Phase 2 & Recruiting & Colorectal Neoplasms \\
\hline NCT00019331 & IL-2 & Phase 2 & Completed & Colorectal Cancer | Colon Cancer \\
\hline NCT00176761 & IL-2 & Phase 2 & Completed & Metastatic Colorectal Cancer \\
\hline NCT00176761 & IL-2 & Phase 2 & Terminated & CRC \\
\hline NCT02919644 & IL-2 & Phase 2 & Active, not recruiting & CRC \\
\hline NCT03222089 & IL-2 & Phase 2 & Withdrawn & CRC \\
\hline 2014-000550-12 & IL-1a & Phase 3 & Completed & $\begin{array}{l}\text { Symptomatic Colorectal Cancer Patients Refractory to Standard } \\
\text { Therapy }\end{array}$ \\
\hline EUCTR2005-003458-81-IT & IL-2 & Phase 3 & Completed & Colorectal Cancer \\
\hline 2005-003458-81 & IL-2 & Phase 3 & Completed & Advanced Colon-Cancer \\
\hline
\end{tabular}

\section{Using in adjuvant therapy}

As the tumorigenesis and development of CRC are complex, there are many factors that may function in these processes. Therefore, creative ideas for the use of ILs as adjuvant therapies are important. DC-based cancer immunotherapy is an effective treatment for stimulating the immune response against tumours. However, maintaining long-term $\mathrm{T}$ cell responses has been problematic. In a phase I clinical study, researchers recruited 12 patients with metastatic CRC, for which conventional treatment had no significant effect. First, carcinoembryonic antigen (CEA)-pulsed DCs and tetanus toxoid were injected into the patients, followed by three injections of CEA-pulsed DCs and a small amount of injected IL-2. The small amount of IL-2 added after the inoculation helped to prolong the $\mathrm{T}$ cell response time. This clinical trial study found that this immunotherapy had a certain clinical significance without obvious side effects and deserved to be further researched [140].

\section{Functioning as clinical indicator}

In addition, ILs can be used as clinical indicators for use in some clinical judgements. In two randomized phase III trials, it was confirmed that the IL-6 rs2069837 genotype could be used as a clinical prognostic factor for mCRC patients who received bevacizumab-based chemotherapy [141]. For the mCRC treatment, health-related quality of life (HRQoL) is a significant outcome. The researchers tested HRQoL levels of $512 \mathrm{mCRC}$ patients receiving cetuximab and found that patients with high serum IL-6 or C-reactive protein (CRP) had worse HRQoL outcomes than patients with normal levels [142]. In another trial, the researchers separated 40 patients with CRC into two groups: using open colectomy as group $\mathrm{A}$ and using laparoscopic colectomy as group B. The researchers measured IL-6, TLR-2, TNF-a, TLR-4 and high-sensitivity CRP (hsCRP) levels and concluded that laparoscopic colectomy had short-term clinical advantages compared with open colectomy owing to the higher levels of IL-6, TLR-2, TLR-4 and hsCRP in group A than compared to those in group B [143].

\section{Conclusion}

The role of cytokines in cancer is fascinating. As a significant component of cytokines, ILs have enlightening roles in CRC. In this review, we have summarized the significant roles of various IL families in CRC as reported in recent years. As new members of ILs which may exert enormous effects on CRC are constantly being discovered, the underlying 
molecule mechanisms of new members on CRC should be given enough attention. In the case of ILs with detailed laboratory research foundation and bioinformation analysis, clinical applications including clinical side effects, efficacy, and combined use need to be investigated. Evidence shows that ILs have a tremendous connection with plentiful cells within the TME, then describing the communication between ILs and individual cells, including immune cells, may have an indelible role in the future. It's interesting that some ILs such as IL-33, IL-21 and IL-10 have exerted dual influence in the CRC. However it is still confusing whether the dual influence is due to experimental limitations or other undiscovered mechanisms. Ingeniously, some intestinal microorganisms can communicate with ILs and ultimately affect CRC. How intestinal microorganisms alter ILs and how to put this into clinical application cannot be missed.

\section{Abbreviations}

ADK: adenylate kinase; CAC: colitis-associated cancer; CC: colon cancer; CCSC: colorectal cancer stem cell; cfDNA: circulating cell-free DNA; CRC: colorectal cancer; DCs: dendritic cells; DOT1L: disruptor of the telomeric silencing 1-like; EAPP: artemisia princeps Pampanini cv; SajabalEMT: epithelial-mesenchymal transition; Hh: helicobacter hepaticus; HRQoL: health-related quality of life; IECs: intestinal epithelial cells; IFP: interstitial fluid pressure; IL-22BP: IL-22-binding protein; ILs: interleukins; iNOS: nitrogen oxide intermediates; MDR1: multidrug resistant 1; NK cells: natural killer cells; PTF: perfusable tissue fraction; SEMFs: subepithelial myofibroblasts; SIGIRR: single immunoglobulin IL-1 receptor-related molecule; TBF: tumour blood flow; TME: the tumour microenvironment.

\section{Acknowledgements}

We thank American Journal Experts (AJE) for their professional English language editing.This study was supported by grants from the Natural Science Foundation of China (no. 81572972), the Supporting Plan of Scientific and Technological Innovation Team in Universities of Henan Province (no. 20IRTSTHN029), the Undergraduate Innovation and Entrepreneurship Training Program (no. 2019cxcy017) and the Research Project on educational and teaching reform of Zhengzhou university (no. 2019ZZUJGLX177).

\section{Author Contributions}

Jingjing Li, Ling Huang, and Jing Lu had the idea for the article. Literature search and data analysis were performed by Jingjing $\mathrm{Li}$, Ling Huang, Hanzhang Zhao, and Yuheng Yan. The manuscript was written by Jingjing Li, Ling Huang, Hanzhang Zhao, and Yuheng Yan. Jing Lu critically revised the work. All authors read and approved the final manuscript.

\section{Competing Interests}

The authors have declared that no competing interest exists.

\section{References}

1. Ferlay J, Soerjomataram I, Dikshit R, Eser S, Mathers C, Rebelo M, et al. Cancer incidence and mortality worldwide: sources, methods and major patterns in GLOBOCAN 2012. International journal of cancer. 2015; 136: E359-86.

2. Brown KGM, Solomon MJ, Mahon K, O'Shannassy S. Management of colorectal cancer. Bmj. 2019; 366: 14561.

3. Ganesh K, Stadler ZK, Cercek A, Mendelsohn RB, Shia J, Segal NH, et al. Immunotherapy in colorectal cancer: rationale, challenges and potential. Nature reviews Gastroenterology \& hepatology. 2019; 16: 361-75.

4. Kopetz S. New therapies and insights into the changing landscape of colorectal cancer. Nature reviews Gastroenterology \& hepatology. 2019; 16: 79-80

5. Ning C, Li YY, Wang Y, Han GC, Wang RX, Xiao H, et al. Complement activation promotes colitis-associated carcinogenesis through activating intestinal IL-1beta/IL-17A axis. Mucosal immunology. 2015; 8: 1275-84.

6. Hai Ping P, Feng Bo T, Li L, Nan Hui Y, Hong Z. IL-1beta/NF-kb signaling promotes colorectal cancer cell growth through miR-181a/PTEN axis. Archives of biochemistry and biophysics. 2016; 604: 20-6.

7. Zhang Y, Davis C, Shah S, Hughes D, Ryan JC, Altomare D, et al. IL-33 promotes growth and liver metastasis of colorectal cancer in mice by remodeling the tumor microenvironment and inducing angiogenesis. Molecular carcinogenesis. 2017; 56: 272-87.

8. Dinarello CA. The history of fever, leukocytic pyrogen and interleukin-1. Temperature. 2015; 2: 8-16.

9. Chen Y, Yang Z, Deng B, Wu D, Quan Y, Min Z. Interleukin 1beta/1RA axis in colorectal cancer regulates tumor invasion, proliferation and apoptosis via autophagy. Oncology reports. 2020; 43: 908-18.

10. Miyoshi H, Morishita A, Tani J, Sakamoto T, Fujita K, Katsura A, et al. Expression profiles of 507 proteins from a biotin label-based antibody array in human colorectal cancer. Oncology reports. 2014; 31: 1277-81.

11. Pastille E, Wasmer MH, Adamczyk A, Vu VP, Mager LF, Phuong NNT, et al. The IL-33/ST2 pathway shapes the regulatory $\mathrm{T}$ cell phenotype to promote intestinal cancer. Mucosal immunology. 2019; 12: 990-1003.

12. Akimoto M, Maruyama R, Takamaru H, Ochiya T, Takenaga K. Soluble IL-33 receptor sST2 inhibits colorectal cancer malignant growth by modifying the tumour microenvironment. Nature communications. 2016; 7: 13589.

13. Grigaitis $P$, Jonusiene V, Zitkute V, Dapkunas J, Dabkeviciene D, Sasnauskiene A. Exogenous interleukin-1alpha signaling negatively impacts acquired chemoresistance and alters cell adhesion molecule expression pattern in colorectal carcinoma cells HCT116. Cytokine. 2019; 114: 38-46.

14. Lubberink M, Golla SS, Jonasson M, Rubin K, Glimelius B, Sorensen J, et al. (15)O-Water PET Study of the Effect of Imatinib, a Selective Platelet-Derived Growth Factor Receptor Inhibitor, Versus Anakinra, an IL-1R Antagonist, on Water-Perfusable Tissue Fraction in Colorectal Cancer Metastases. Journal of nuclear medicine : official publication, Society of Nuclear Medicine. 2015; 56: 1144-9.

15. Gelfo V, Mazzeschi M, Grilli G, Lindzen M, Santi S, D'Uva G, et al. A Novel Role for the Interleukin-1 Receptor Axis in Resistance to Anti-EGFR Therapy. Cancers. 2018; 10.

16. Zhao J, Bulek K, Gulen MF, Zepp JA, Karagkounis G, Martin BN, et al. Human Colon Tumors Express a Dominant-Negative Form of SIGIRR That Promotes Inflammation and Colitis-Associated Colon Cancer in Mice. Gastroenterology. 2015; 149: 1860-71 e8

17. Mar AC, Chu CH, Lee HJ, Chien CW, Cheng JJ, Yang SH, et al. Interleukin-1 Receptor Type 2 Acts with c-Fos to Enhance the Expression of Interleukin-6 and Vascular Endothelial Growth Factor A in Colon Cancer Cells and Induce Angiogenesis. The Journal of biological chemistry. 2015; 290: 22212-24.

18. He C, Yu T, Shi Y, Ma C, Yang W, Fang L, et al. MicroRNA 301A Promotes Intestinal Inflammation and Colitis-Associated Cancer Development by Inhibiting BTG1. Gastroenterology. 2017; 152: 1434-48 e15.

19. Yao J, Hu R, Sun J, Lin B, Zhao L, Sha Y, et al. Oroxylin A prevents inflammation-related tumor through down-regulation of inflammatory gene expression by inhibiting NF-kappaB signaling. Molecular carcinogenesis. 2014; 53: 145-58

20. Wang Y, Lu P, Zhang W, Du Q, Tang J, Wang H, et al. GEN-27, a Newly Synthetic Isoflavonoid, Inhibits the Proliferation of Colon Cancer Cells in 
Inflammation Microenvironment by Suppressing NF-kappaB Pathway. Mediators of inflammation. 2016; 2016: 2853040.

21. Kim YH, Kim JH, Kim BG, Lee KL, Kim JW, Koh SJ. Tauroursodeoxycholic acid attenuates colitis-associated colon cancer by inhibiting nuclear factor kappaB signaling. Journal of gastroenterology and hepatology. 2019; 34: 544-51.

22. Chung KS, Choi HE, Shin JS, Cho EJ, Cho YW, Choi JH, et al. Chemopreventive effects of standardized ethanol extract from the aerial parts of Artemisia princeps Pampanini cv. Sajabal via NF-kappaB inactivation on colitis-associated colon tumorigenesis in mice. Food and chemical toxicology : an international journal published for the British Industrial Biological Research Association. 2015; 75: 14-23.

23. Sconocchia G, Eppenberger-Castori S, Zlobec I, Karamitopoulou E, Arriga R, Coppola A, et al. HLA class II antigen expression in colorectal carcinoma tumors as a favorable prognostic marker. Neoplasia. 2014; 16: 31-42.

24. Wang Y, Wang K, Han GC, Wang RX, Xiao H, Hou CM, et al. Neutrophil infiltration favors colitis-associated tumorigenesis by activating the interleukin-1 (IL-1)/IL-6 axis. Mucosal immunology. 2014; 7: 1106-15.

25. Bergman M, Levin GS, Bessler H, Djaldetti M, Salman H. Resveratrol affects the cross talk between immune and colon cancer cells. Biomedicine \& pharmacotherapy $=$ Biomedecine \& pharmacotherapie. 2013; 67: 43-7.

26. Li $\mathrm{Q}$, Zhang $\mathrm{X}$, Wang $\mathrm{W}$, Li $\mathrm{L}, \mathrm{Xu} \mathrm{Q}, \mathrm{Wu} X$, et al. CPT-11 activates NLRP3 inflammasome through JNK and NF-kappaB signalings. Toxicology and applied pharmacology. 2015; 289: 133-41

27. Guabiraba R, Besnard AG, Menezes GB, Secher T, Jabir MS, Amaral SS, et al. IL-33 targeting attenuates intestinal mucositis and enhances effective tumor chemotherapy in mice. Mucosal immunology. 2014; 7: 1079-93.

28. Guo W, Sun Y, Liu W, Wu X, Guo L, Cai P, et al. Small molecule-driven mitophagy-mediated NLRP3 inflammasome inhibition is responsible for the prevention of colitis-associated cancer. Autophagy. 2014; 10: 972-85.

29. Dupaul-Chicoine J, Arabzadeh A, Dagenais M, Douglas T, Champagne C, Morizot A, et al. The Nlrp3 Inflammasome Suppresses Colorectal Cancer Metastatic Growth in the Liver by Promoting Natural Killer Cell Tumoricidal Activity. Immunity. 2015; 43: 751-63.

30. Sharma D, Malik A, Guy CS, Karki R, Vogel P, Kanneganti TD. Pyrin Inflammasome Regulates Tight Junction Integrity to Restrict Colitis and Tumorigenesis. Gastroenterology. 2018; 154: 948-64 e8.

31. Malik A, Sharma D, Malireddi RKS, Guy CS, Chang TC, Olsen SR, et al. SYK-CARD9 Signaling Axis Promotes Gut Fungi-Mediated Inflammasome Activation to Restrict Colitis and Colon Cancer. Immunity. 2018; 49: 515-30 e5.

32. Higashi K, Hazama S, Araki A, Yoshimura K, Iizuka N, Yoshino S, et al. A novel cancer vaccine strategy with combined IL-18 and HSV-TK gene therapy driven by the hTERT promoter in a murine colorectal cancer model. International journal of oncology. 2014; 45: 1412-20.

33. Yan X, Zhao J, Zhang R. Interleukin-37 mediates the antitumor activity in colon cancer through beta-catenin suppression. Oncotarget. 2017; 8: 49064-75.

34. Weinstein AM, Giraldo NA, Petitprez F, Julie C, Lacroix L, Peschaud F, et al. Association of IL-36gamma with tertiary lymphoid structures and inflammatory immune infiltrates in human colorectal cancer. Cancer immunology, immunotherapy : CII. 2019; 68: 109-20.

35. Zhu B, Luo J, Jiang Y, Yu L, Liu M, Fu J. Prognostic significance of nomograms integrating IL-37 expression, neutrophil level, and MMR status in patients with colorectal cancer. Cancer medicine. 2018; 7: 3682-94.

36. Fang M, Li Y, Huang K, Qi S, Zhang J, Zgodzinski W, et al. IL33 Promotes Colon Cancer Cell Stemness via JNK Activation and Macrophage Recruitment. Cancer research. 2017; 77: 2735-45.

37. Maywald RL, Doerner SK, Pastorelli L, De Salvo C, Benton SM, Dawson EP, et al. IL-33 activates tumor stroma to promote intestinal polyposis. Proceedings of the National Academy of Sciences of the United States of America. 2015; 112: E2487-96

38. Cui G, Yuan A, Li Z, Goll R, Florholmen J. ST2 and regulatory T cells in the colorectal adenoma/carcinoma microenvironment: implications for diseases progression and prognosis. Scientific reports. 2020; 10: 5892.

39. Eissmann MF, Dijkstra C, Wouters MA, Baloyan D, Mouradov D, Nguyen PM, et al. Interleukin 33 Signaling Restrains Sporadic Colon Cancer in an Interferon-gamma-Dependent Manner. Cancer immunology research. 2018; 6: 409-21.

40. Malik A, Sharma D, Zhu Q, Karki R, Guy CS, Vogel P, et al. IL-33 regulates the IgA-microbiota axis to restrain IL-1alpha-dependent colitis and tumorigenesis. The Journal of clinical investigation. 2016; 126: 4469-81.

41. Morgan DA, Ruscetti FW, Gallo R. Selective in vitro growth of T lymphocytes from normal human bone marrows. Science. 1976; 193: 1007-8.

42. Leonard WJ, Lin JX, O'Shea JJ. The gammac Family of Cytokines: Basic Biology to Therapeutic Ramifications. Immunity. 2019; 50: 832-50.

43. Dimberg J, Shamoun L, Landerholm K, Andersson RE, Kolodziej B, Wagsater D. Genetic Variants of the IL2 Gene Related to Risk and Survival in Patients With Colorectal Cancer. Anticancer research. 2019; 39: 4933-40.

44. Rocca YS, Roberti MP, Julia EP, Pampena MB, Bruno L, Rivero S, et al. Phenotypic and Functional Dysregulated Blood NK Cells in Colorectal Cancer Patients Can Be Activated by Cetuximab Plus IL-2 or IL-15. Frontiers in immunology. 2016; 7: 413 .

45. Wang C, Lu Y, Chen L, Gao T, Yang Q, Zhu C, et al. Th9 cells are subjected to PD-1/PD-L1-mediated inhibition and are capable of promoting CD8 T cell expansion through IL-9R in colorectal cancer. International immunopharmacology. 2020; 78: 106019.
46. Liu X, Li Y, Sun X, Muftuoglu Y, Wang B, Yu T, et al. Powerful anti-colon cancer effect of modified nanoparticle-mediated IL-15 immunogene therapy through activation of the host immune system. Theranostics. 2018; 8: 3490-503.

47. Cui G, Yuan A, Zhu L, Florholmen J, Goll R. Increased expression of interleukin-21 along colorectal adenoma-carcinoma sequence and its predicating significance in patients with sporadic colorectal cancer. Clinical immunology. 2017; 183: 266-72.

48. Shi W, Dong L, Sun Q, Ding H, Meng J, Dai G. Follicular helper T cells promote the effector functions of $\mathrm{CD} 8(+) \mathrm{T}$ cells via the provision of IL-21, which is downregulated due to PD-1/PD-L1-mediated suppression in colorectal cancer. Experimental cell research. 2018; 372: 35-42.

49. Bednarz-Misa I, Diakowska D, Krzystek-Korpacka M. Local and Systemic IL-7 Concentration in Gastrointestinal-Tract Cancers. Medicina. 2019; 55.

50. Krzystek-Korpacka M, Zawadzki M, Neubauer K, Bednarz-Misa I, Gorska S, Wisniewski J, et al. Elevated systemic interleukin-7 in patients with colorectal cancer and individuals at high risk of cancer: association with lymph node involvement and tumor location in the right colon. Cancer immunology, immunotherapy : CII. 2017; 66: 171-9.

51. Chen J, Gong C, Mao H, Li Z, Fang Z, Chen Q, et al. E2F1/SP3/STAT6 axis is required for IL-4-induced epithelial-mesenchymal transition of colorectal cancer cells. International journal of oncology. 2018; 53: 567-78

52. Lin $X$, Wang $S$, Sun $M$, Zhang $C$, Wei $C$, Yang $C$, et al. miR-195-5p/NOTCH2-mediated EMT modulates IL-4 secretion in colorectal cancer to affect M2-like TAM polarization. Journal of hematology \& oncology. 2019; 12: 20.

53. Tepper RI, Pattengale PK, Leder P. Murine interleukin-4 displays potent anti-tumor activity in vivo. Cell. 1989; 57: 503-12.

54. Mackall CL, Fry TJ, Gress RE. Harnessing the biology of IL-7 for therapeutic application. Nature reviews Immunology. 2011; 11: 330-42.

55. Sportes C, Babb RR, Krumlauf MC, Hakim FT, Steinberg SM, Chow CK, et al. Phase I study of recombinant human interleukin-7 administration in subjects with refractory malignancy. Clinical cancer research : an official journal of the American Association for Cancer Research. 2010; 16: 727-35.

56. Waldmann TA. The biology of interleukin-2 and interleukin-15: implications for cancer therapy and vaccine design. Nature reviews Immunology. 2006; 6: 595-601.

57. Noelle RJ, Nowak EC. Cellular sources and immune functions of interleukin-9. Nature reviews Immunology. 2010; 10: 683-7.

58. Wang J, Sun M, Zhao H, Huang Y, Li D, Mao D, et al. IL-9 Exerts Antitumor Effects in Colon Cancer and Transforms the Tumor Microenvironment In vivo. Technology in cancer research \& treatment. 2019; 18: 1533033819857737.

59. Li Y, Qiu Q, Fan Z, He P, Chen H, Jiao X. Th17 cytokine profiling of colorectal cancer patients with or without enterovirus 71 antigen expression. Cytokine. 2018; 107: 35-42.

60. De Simone V, Franze E, Ronchetti G, Colantoni A, Fantini MC, Di Fusco D, et al. Th17-type cytokines, IL-6 and TNF-alpha synergistically activate STAT3 and NF-kB to promote colorectal cancer cell growth. Oncogene. 2015; 34: 3493-503.

61. De Simone V, Pallone F, Monteleone G, Stolfi C. Role of TH17 cytokines in the control of colorectal cancer. Oncoimmunology. 2013; 2: e26617.

62. Jones SA, Jenkins BJ. Recent insights into targeting the IL-6 cytokine family in inflammatory diseases and cancer. Nature reviews Immunology. 2018; 18: 773-89.

63. Bazan JF. Haemopoietic receptors and helical cytokines. Immunology today. 1990; 11: 350-4.

64. Bazan JF. A novel family of growth factor receptors: a common binding domain in the growth hormone, prolactin, erythropoietin and IL-6 receptors, and the p75 IL-2 receptor beta-chain. Biochemical and biophysical research communications. 1989; 164: 788-95.

65. Rose-John S. Interleukin-6 Family Cytokines. Cold Spring Harbor perspectives in biology. 2018; 10.

66. Luo Y, Wu X, Ma Z, Tan W, Wang L, Na D, et al. Expression of the novel adipokine C1qTNF-related protein 4 (CTRP4) suppresses colitis and colitis-associated colorectal cancer in mice. Cellular \& molecular immunology. 2016; 13: 688-99

67. Grivennikov SI, Karin M. Inflammatory cytokines in cancer: tumour necrosis factor and interleukin 6 take the stage. Annals of the rheumatic diseases. 2011; 70 Suppl 1: i104-8

68. Zhu L, Cheng $\mathrm{X}$, Ding $\mathrm{Y}$, Shi $\mathrm{J}$, Jin $\mathrm{H}$, Wang $\mathrm{H}$, et al. Bone marrow-derived myofibroblasts promote colon tumorigenesis through the IL-6/JAK2/STAT3 pathway. Cancer letters. 2014; 343: 80-9.

69. Yokoigawa N, Takeuchi N, Toda M, Inoue M, Kaibori M, Yanagida H, et al. Enhanced production of interleukin 6 in peripheral blood monocytes stimulated with mucins secreted into the bloodstream. Clinical cancer research: an official journal of the American Association for Cancer Research. 2005; 11: 6127-32.

70. Heichler C, Scheibe K, Schmied A, Geppert CI, Schmid B, Wirtz S, et al. STAT3 activation through IL-6/IL-11 in cancer-associated fibroblasts promotes colorectal tumour development and correlates with poor prognosis. Gut. 2019.

71. Jiang F, Liu M, Wang H, Shi G, Chen B, Chen T, et al. Wu Mei Wan attenuates CAC by regulating gut microbiota and the NF-kB/IL6-STAT3 signaling pathway. Biomedicine \& pharmacotherapy $=$ Biomedecine \& pharmacotherapie. 2020; 125: 109982.

72. Li ZW, Sun B, Gong T, Guo S, Zhang J, Wang J, et al. GNAI1 and GNAI3 Reduce Colitis-Associated Tumorigenesis in Mice by Blocking IL6 Signaling 
and Down-regulating Expression of GNAI2. Gastroenterology. 2019; 156: 2297-312.

73. Gao S, Hu J, Wu X, Liang Z. PMA treated THP-1-derived-IL-6 promotes EMT of SW48 through STAT3/ERK-dependent activation of Wnt/beta-catenin signaling pathway. Biomedicine \& pharmacotherapy $=$ Biomedecine \& pharmacotherapie. 2018; 108: 618-24.

74. Rokavec M, Oner MG, Li H, Jackstadt $\mathrm{R}$, Jiang $\mathrm{L}$, Lodygin $\mathrm{D}$, et al. IL-6R/STAT3/miR-34a feedback loop promotes EMT-mediated colorectal cancer invasion and metastasis. The Journal of clinical investigation. 2014; 124: 1853-67.

75. Li YY, Hsieh LL, Tang RP, Liao SK, Yeh KY. Interleukin-6 (IL-6) released by macrophages induces IL-6 secretion in the human colon cancer HT-29 cell line. Human immunology. 2009; 70: 151-8.

76. Xu K, Zhan Y, Yuan Z, Qiu Y, Wang H, Fan G, et al. Hypoxia Induces Drug Resistance in Colorectal Cancer through the HIF-1alpha/miR-338-5p/IL-6 Feedback Loop. Molecular therapy : the journal of the American Society of Gene Therapy. 2019; 27: 1810-24.

77. Nagasaki T, Hara $M$, Nakanishi $H$, Takahashi $H$, Sato $M$, Takeyama H. Interleukin-6 released by colon cancer-associated fibroblasts is critical for tumour angiogenesis: anti-interleukin-6 receptor antibody suppressed angiogenesis and inhibited tumour-stroma interaction. British journal of cancer. 2014; 110: 469-78.

78. Ye X, Wu H, Sheng L, Liu YX, Ye F, Wang M, et al. Oncogenic potential of truncated RXRalpha during colitis-associated colorectal tumorigenesis by promoting IL-6-STAT3 signaling. Nature communications. 2019; 10: 1463.

79. Wei J, Ma L, Lai YH, Zhang R, Li H, Li C, et al. Bazedoxifene as a novel GP130 inhibitor for Colon Cancer therapy. Journal of experimental \& clinical cancer research : CR. 2019; 38: 63.

80. Curtis DJ, Hilton DJ, Roberts B, Murray L, Nicola N, Begley CG. Recombinant soluble interleukin-11 (IL-11) receptor alpha-chain can act as an IL-11 antagonist. Blood. 1997; 90: 4403-12.

81. Heinrich PC, Behrmann I, Muller-Newen G, Schaper F, Graeve L. Interleukin-6-type cytokine signalling through the gp130/Jak/STAT pathway. The Biochemical journal. 1998; 334 ( Pt 2): 297-314.

82. Yoshizaki A, Nakayama T, Yamazumi K, Yakata Y, Taba M, Sekine I, Expression of interleukin (IL)-11 and IL-11 receptor in human colorectal adenocarcinoma: IL-11 up-regulation of the invasive and proliferative activity of human colorectal carcinoma cells. International journal of oncology. 2006; 29: 869-76.

83. Shi C, Yang Y, Xia Y, Okugawa Y, Yang J, Liang Y, et al. Novel evidence for an oncogenic role of microRNA-21 in colitis-associated colorectal cancer. Gut. 2016; 65: 1470-81.

84. Wolff C, Zoschke C, Kalangi SK, Reddanna P, Schafer-Korting M. Tumor microenvironment determines drug efficacy in vitro - apoptotic and anti-inflammatory effects of 15-lipoxygenase metabolite, 13-HpOTrE. European journal of pharmaceutics and biopharmaceutics : official journal of Arbeitsgemeinschaft fur Pharmazeutische Verfahrenstechnik eV. 2019; 142: $1-7$.

85. Kang S, Kim BR, Kang MH, Kim DY, Lee DH, Oh SC, et al. Anti-metastatic effect of metformin via repression of interleukin 6-induced epithelial-mesenchymal transition in human colon cancer cells. PloS one. 2018; 13: e0205449.

86. Wawro ME, Sobierajska K, Ciszewski WM, Niewiarowska J. Nonsteroidal Anti-Inflammatory Drugs Prevent Vincristine-Dependent Cancer-Associated Fibroblasts Formation. International journal of molecular sciences. 2019; 20.

87. Liu Q, Li A, Tian Y, Wu JD, Liu Y, Li T, et al. The CXCL8-CXCR1/2 pathways in cancer. Cytokine \& growth factor reviews. 2016; 31: 61-71

88. Xia W, Chen W, Zhang Z, Wu D, Wu P, Chen Z, et al. Prognostic value, clinicopathologic features and diagnostic accuracy of interleukin-8 in colorectal cancer: a meta-analysis. PloS one. 2015; 10: e0123484.

89. Xiao YC, Yang ZB, Cheng XS, Fang XB, Shen T, Xia CF, et al. CXCL8, overexpressed in colorectal cancer, enhances the resistance of colorectal cancer cells to anoikis. Cancer letters. 2015; 361: 22-32.

90. Jin WJ, Xu JM, Xu WL, Gu DH, Li PW. Diagnostic value of interleukin-8 in colorectal cancer: a case-control study and meta-analysis. World journal of gastroenterology. 2014; 20: 16334-42.

91. Dabkeviciene D, Jonusiene V, Zitkute V, Zalyte E, Grigaitis P, Kirveliene V, et al. The role of interleukin-8 (CXCL8) and CXCR2 in acquired chemoresistance of human colorectal carcinoma cells HCT116. Medical oncology. 2015; 32: 258 .

92. Shen T, Yang Z, Cheng X, Xiao Y, Yu K, Cai X, et al. CXCL8 induces epithelial-mesenchymal transition in colon cancer cells via the PI3K/Akt/NF-kappaB signaling pathway. Oncology reports. 2017; 37: 2095-100.

93. Du J, He Y, Li P, Wu W, Chen Y, Ruan H. IL-8 regulates the doxorubicin resistance of colorectal cancer cells via modulation of multidrug resistance 1 (MDR1). Cancer chemotherapy and pharmacology. 2018; 81: 1111-9.

94. Fisher RC, Bellamkonda K, Alex Molina L, Xiang S, Liska D, Sarvestani SK, et al. Disrupting Inflammation-Associated CXCL8-CXCR1 Signaling Inhibits Tumorigenicity Initiated by Sporadic- and Colitis-Colon Cancer Stem Cells. Neoplasia. 2019; 21: 269-81

95. Jin G, Yang Y, Liu K, Zhao J, Chen X, Liu H, et al. Combination curcumin and (-)-epigallocatechin-3-gallate inhibits colorectal carcinoma microenvironment-induced angiogenesis by JAK/STAT3/IL-8 pathway. Oncogenesis. 2017; 6: e384
96. Wei H, Li J, Xie M, Lei R, Hu B. Comprehensive analysis of metastasis-related genes reveals a gene signature predicting the survival of colon cancer patients. PeerJ. 2018; 6: e5433.

97. Cheng XS, Li YF, Tan J, Sun B, Xiao YC, Fang XB, et al. CCL20 and CXCL8 synergize to promote progression and poor survival outcome in patients with colorectal cancer by collaborative induction of the epithelial-mesenchymal transition. Cancer letters. 2014; 348: 77-87.

98. Sun Q, Sun F, Wang B, Liu S, Niu W, Liu E, et al. Interleukin-8 promotes cell migration through integrin alphavbeta6 upregulation in colorectal cancer. Cancer letters. 2014; 354: 245-53.

99. Shimizu M, Tanaka N. IL-8-induced O-GlcNAc modification via GLUT3 and GFAT regulates cancer stem cell-like properties in colon and lung cancer cells. Oncogene. 2019; 38: 1520-33.

100. Ibusuki $K$, Sakiyama $T$, Kanmura $S$, Maeda $T$, Iwashita $Y$, Nasu $Y$, et al. Human neutrophil peptides induce interleukin-8 in intestinal epithelial cells through the P2 receptor and ERK1/2 signaling pathways. International journal of molecular medicine. 2015; 35: 1603-9.

101. Niu Z, Tang W, Liu T, Xu P, Zhu D, Ji M, et al. Cell-free DNA derived from cancer cells facilitates tumor malignancy through Toll-like receptor 9 signaling-triggered interleukin-8 secretion in colorectal cancer. Acta biochimica et biophysica Sinica. 2018; 50: 1007-17.

102. Bahrami F, Kukulski F, Lecka J, Tremblay A, Pelletier J, Rockenbach L, et al. Purine-metabolizing ectoenzymes control IL-8 production in human colon HT-29 cells. Mediators of inflammation. 2014; 2014: 879895.

103. Geng R, Tan X, Wu J, Pan Z, Yi M, Shi W, et al. RNF183 promotes proliferation and metastasis of colorectal cancer cells via activation of NF-kap paB-IL-8 axis. Cell death \& disease. 2017; 8: e2994.

104. Kano S, Nishida K, Kurebe H, Nishiyama C, Kita K, Akaike Y, et al. Oxidative stress-inducible truncated serine/arginine-rich splicing factor 3 reg ulates interleukin-8 production in human colon cancer cells. American journal of physiology Cell physiology. 2014; 306: C250-62

105. Shuai F, Wang B, Dong S. MicroRNA-204 Inhibits the Growth and Motility of Colorectal Cancer Cells by Downregulation of CXCL8. Oncology research. 2018; 26: 1295-305.

106. Lin SC, Hsiao KY, Chang N, Hou PC, Tsai SJ. Loss of dual-specificity phosphatase- 2 promotes angiogenesis and metastasis via up-regulation of interleukin-8 in colon cancer. The Journal of pathology. 2017; 241: 638-48.

107. Ouyang W, O'Garra A. IL-10 Family Cytokines IL-10 and IL-22: from Basic Science to Clinical Translation. Immunity. 2019; 50: 871-91.

108. Moore KW, de Waal Malefyt R, Coffman RL, O'Garra A. Interleukin-10 and the interleukin-10 receptor. Annual review of immunology. 2001; 19: 683-765.

109. Xie MH, Aggarwal S, Ho WH, Foster J, Zhang Z, Stinson J, et al. Interleukin (IL)-22, a novel human cytokine that signals through the interferon receptor-related proteins CRF2-4 and IL-22R. The Journal of biological chemistry. 2000; 275 : 31335-9.

110. Dumoutier L, Van Roost E, Colau D, Renauld JC. Human interleukin-10-related T cell-derived inducible factor: molecular cloning and functional characterization as an hepatocyte-stimulating factor. Proceedings of the National Academy of Sciences of the United States of America. 2000; 97: 10144-9.

111. Dumoutier L, Lejeune D, Colau D, Renauld JC. Cloning and characterization of IL-22 binding protein, a natural antagonist of IL-10-related T cell-derived inducible factor/IL-22. Journal of immunology. 2001; 166: 7090-5.

112. Qi L, Ding Y. Screening of Differentiation-Specific Molecular Biomarkers for Colon Cancer. Cellular physiology and biochemistry : international journal of experimental cellular physiology, biochemistry, and pharmacology. 2018; 46: 2543-50

113. Doulabi $H$, Rastin $M$, Shabahangh $H$, Maddah $G$, Abdollahi A, Nosratabadi $R$, et al. Analysis of Th22, Th17 and CD4(+)cells co-producing IL-17/IL-22 at different stages of human colon cancer. Biomedicine \& pharmacotherapy $=$ Biomedecine \& pharmacotherapie. 2018; 103: 1101-6.

114. Wang C, Gong G, Sheh A, Muthupalani S, Bryant EM, Puglisi DA, et al Interleukin-22 drives nitric oxide-dependent DNA damage and dysplasia in a murine model of colitis-associated cancer. Mucosal immunology. 2017; 10: 1504-17.

115. Ziesche $\mathrm{E}$, Bachmann $\mathrm{M}$, Kleinert $\mathrm{H}$, Pfeilschifter $\mathrm{J}$, Muhl $\mathrm{H}$. The interleukin-22/STAT3 pathway potentiates expression of inducible nitric-oxide synthase in human colon carcinoma cells. The Journal of biological chemistry. 2007; 282: 16006-15.

116. Fukui H, Sekikawa A, Tanaka H, Fujimori Y, Katake Y, Fujii S, et al. DMBT1 is a novel gene induced by IL-22 in ulcerative colitis. Inflammatory bowel diseases. 2011: 17: 1177-88.

117. Moniruzzaman M, Wang R, Jeet V, McGuckin MA, Hasnain SZ. Interleukin (IL)-22 from IL-20 Subfamily of Cytokines Induces Colonic Epithelial Cell Proliferation Predominantly through ERK1/2 Pathway. International journal of molecular sciences. 2019; 20 .

118. Huang YH, Cao YF, Jiang ZY, Zhang S, Gao F. Th22 cell accumulation is associated with colorectal cancer development. World journal of gastroenterology. 2015; 21: 4216-24

119. Kryczek I, Lin Y, Nagarsheth N, Peng D, Zhao L, Zhao E, et al. IL-22(+)CD4(+) $\mathrm{T}$ cells promote colorectal cancer stemness via STAT3 transcription factor activation and induction of the methyltransferase DOT1L. Immunity. 2014; 40: $772-84$ 
120. Zhang R, Men K, Zhang X, Huang R, Tian Y, Zhou B, et al. Delivery of a Modified mRNA Encoding IL-22 Binding Protein (IL-22BP) for Colon Cancer Gene Therapy. Journal of biomedical nanotechnology. 2018; 14: 1239-51.

121. Ho AS, Liu Y, Khan TA, Hsu DH, Bazan JF, Moore KW. A receptor for interleukin 10 is related to interferon receptors. Proceedings of the National Academy of Sciences of the United States of America. 1993; 90: 11267-71.

122. Kotenko SV, Krause CD, Izotova LS, Pollack BP, Wu W, Pestka S. Identification and functional characterization of a second chain of the interleukin-10 receptor complex. The EMBO journal. 1997; 16: 5894-903.

123. Shouval DS, Biswas A, Goettel JA, McCann K, Conaway E, Redhu NS, et al. Interleukin-10 receptor signaling in innate immune cells regulates mucosal immune tolerance and anti-inflammatory macrophage function. Immunity. 2014; 40: 706-19.

124. Rossowska J, Anger N, Szczygiel A, Mierzejewska J, Pajtasz-Piasecka E. Reprogramming the murine colon cancer microenvironment using lentivectors encoding shRNA against IL-10 as a component of a potent DC-based chemoimmunotherapy. Journal of experimental \& clinical cancer research: CR. 2018; 37: 126

125. Del Carmen S, de Moreno de LeBlanc A, Levit R, Azevedo V, Langella P, Bermudez-Humaran LG, et al. Anti-cancer effect of lactic acid bacteria expressing antioxidant enzymes or IL-10 in a colorectal cancer mouse model. International immunopharmacology. 2017; 42: 122-9.

126. Li B, Wang F, Ma C, Hao T, Geng L, Jiang H. Predictive value of IL-18 and IL-10 in the prognosis of patients with colorectal cancer. Oncology letters. 2019; 18: 713-9.

127. Moseley TA, Haudenschild DR, Rose L, Reddi AH. Interleukin-17 family and IL-17 receptors. Cytokine \& growth factor reviews. 2003; 14: 155-74.

128. Iwakura $\mathrm{Y}$, Ishigame $\mathrm{H}$, Saijo $\mathrm{S}$, Nakae S. Functional specialization of interleukin-17 family members. Immunity. 2011; 34: 149-62.

129. Amicarella F, Muraro MG, Hirt C, Cremonesi E, Padovan E, Mele V, et al. Dual role of tumour-infiltrating $\mathrm{T}$ helper 17 cells in human colorectal cancer. Gut. 2017; 66: 692-704.

130. Hurtado CG, Wan F, Housseau F, Sears CL. Roles for Interleukin 17 and Adaptive Immunity in Pathogenesis of Colorectal Cancer. Gastroenterology. 2018; 155: 1706-15.

131. Do Thi VA, Park SM, Lee H, Kim YS. The Membrane-Bound Form of IL-17A Promotes the Growth and Tumorigenicity of Colon Cancer Cells. Molecules and cells. 2016; 39: 536-42.

132. Liao Y, Zhao J, Bulek K, Tang F, Chen X, Cai G, et al. Inflammation mobilizes copper metabolism to promote colon tumorigenesis via an IL-17-STEAP4-XIAP axis. Nature communications. 2020; 11: 900.

133. Chung AS, Wu X, Zhuang G, Ngu H, Kasman I, Zhang I, et al. An interleukin-17-mediated paracrine network promotes tumor resistance to anti-angiogenic therapy. Nature medicine. 2013; 19: 1114-23.

134. Tong Z, Yang XO, Yan H, Liu W, Niu X, Shi Y, et al. A protective role by interleukin-17F in colon tumorigenesis. PloS one. 2012; 7: e34959.

135. Tosolini M, Kirilovsky A, Mlecnik B, Fredriksen T, Mauger S, Bindea G, et al. Clinical impact of different classes of infiltrating $\mathrm{T}$ cytotoxic and helper cells (Th1, th2, treg, th17) in patients with colorectal cancer. Cancer research. 2011; 71: $1263-71$.

136. Naing A, Papadopoulos KP, Autio KA, Ott PA, Patel MR, Wong DJ, et al. Safety, Antitumor Activity, and Immune Activation of Pegylated Recombinant Human Interleukin-10 (AM0010) in Patients With Advanced Solid tumours. Journal of clinical oncology : official journal of the American Society of Clinical Oncology. 2016; 34: 3562-9.

137. Hickish T, Andre T, Wyrwicz L, Saunders M, Sarosiek T, Kocsis I, et al. MABp1 as a novel antibody treatment for advanced colorectal cancer: a randomised, double-blind, placebo-controlled, phase 3 study. The Lancet Oncology. 2017; 18: 192-201.

138. Bilusic M, Heery CR, Collins JM, Donahue RN, Palena C, Madan RA, et al. Phase I trial of HuMax-IL8 (BMS-986253), an anti-IL-8 monoclonal antibody, in patients with metastatic or unresectable solid tumours. Journal for immunotherapy of cancer. 2019; 7: 240.

139. Angevin E, Tabernero J, Elez E, Cohen SJ, Bahleda R, van Laethem JL, et al. A phase I/II, multiple-dose, dose-escalation study of siltuximab, an anti-interleukin-6 monoclonal antibody, in patients with advanced solid tumours. Clinical cancer research : an official journal of the American Association for Cancer Research. 2014; 20: 2192-204.

140. Liu KJ, Chao TY, Chang JY, Cheng AL, Ch'ang HJ, Kao WY, et al. A phase I clinical study of immunotherapy for advanced colorectal cancers using carcinoembryonic antigen-pulsed dendritic cells mixed with tetanus toxoid and subsequent IL-2 treatment. Journal of biomedical science. 2016; 23: 64.

141. Matsusaka S, Hanna DL, Cao S, Zhang W, Yang D, Ning Y, et al. Prognostic Impact of IL6 Genetic Variants in Patients with Metastatic Colorectal Cancer Treated with Bevacizumab-Based Chemotherapy. Clinical cancer research : an official journal of the American Association for Cancer Research. 2016; 22: 3218-26.

142. Thomsen M, Guren MG, Skovlund E, Glimelius B, Hjermstad MJ, Johansen JS, et al. Health-related quality of life in patients with metastatic colorectal cancer, association with systemic inflammatory response and RAS and BRAF mutation status. European journal of cancer. 2017; 81: 26-35.

143. Tsimogiannis KE, Tellis CC, Tselepis AD, Pappas-Gogos GK, Tsimoyiannis EC, Basdanis G. Toll-like receptors in the inflammatory response during open and laparoscopic colectomy for colorectal cancer. Surgical endoscopy. 2012; 26: $330-6$. 